\title{
EEG-BOLD Correlations During (Post-)Adolescent Brain Maturation
}

\author{
Published as: Lüchinger R, Michels L, Martin E, Brandeis D, (2011) EEG-BOLD correlations \\ during (post-) adolescent brain maturation. Neuroimage, 56, 1493-1505.
}

Abstract - The transition from adolescence to adulthood is a critical stage in the human lifespan during which the brain still undergoes substantial structural and functional change. The changing frequency composition of the resting state EEG reflects maturation of brain function. This study investigated (post)adolescent brain maturation captured by two independently but simultaneously recorded neuronal signals: EEG and fMRI. Data were collected in a 20 min eyes-open/eyes-closed resting state paradigm. EEG, fMRI-BOLD signal and EEG-BOLD correlations were compared between groups of adults, age $25(n=18)$, and adolescents, age $15(n=18)$. A typical developmental decrease of low-frequency EEG power was observed even at this late stage of brain maturation. Frequency and condition specific EEG-fMRI correlations proved robust for multiple brain regions. However, no consistent change in the EEG-BOLD correlations was identified that would correspond to the neuronal maturation captured by the EEG. This result indicates that the EEG-BOLD correlation measures a distinct aspect of neurophysiological activity that presumably matures earlier, since it is less sensitive to late maturation than the neuronal activity captured by low-frequency EEG.

\section{Introduction}

Adolescence and the transition into adulthood are critical stages in the human lifespan. The typical emergence of some major mental illnesses during adolescence further indicates fundamental maturational reorganization (Paus et al., 2008) and stresses the importance of understanding late brain maturation. Since the major developmental changes occur during infancy and childhood, specific research on late maturation has often been neglected. However, late maturation, during and after adolescence has attracted increasing attention in recent years (Blakemore and Choudhury, 2006; Sisk and Foster, 2004). A growing body of evidence suggests that there are still substantial structural (Paus, 2005) and functional (Luna et al., 2010) changes in the brain during this time. Gray matter loss and white matter increase in adolescence were found to be consistent with the basic processes 
of neuronal maturation, i.e. synaptic pruning and myelination (Giedd et al., 1999; Gogtay et al., 2004; Sowell et al., 2001). Apart from these structural aspects, executive functioning commonly associated with changes in the prefrontal cortex undergoes late brain maturation (Luna et al., 2010; Stevens, 2009). Recent brain imaging research has also investigated brain function at rest (where no stimulus or task is involved) to identify resting state networks (RSNs) of functional connectivity (Beckmann et al., 2005; Damoiseaux et al., 2006; van den Heuvel and Hulshoff Pol, 2010). In terms of the development of functional connectivity, basic principles such as successive neural segregation and hierarchical organization have been described (Fair et al., 2007; Stevens, 2009).

Converging evidence for late brain maturation comes from electroencephalogram (EEG) research. The EEG noninvasively measures electrical mass activity of neurons. An advantage over other functional methods such as fMRI is that the EEG measures neuronal activity directly and in absolute terms, i.e. in physical units (microvolts, $\mu \mathrm{V}$ ). Consequently EEG research has focused on resting state brain functioning since its discovery (Berger, 1929 ) long before the advent of fMRI. The resting EEG is typically characterized by oscillations of different amplitudes and frequencies. One of the major findings since the beginning of resting EEG research is that children's EEG is dominated by slower rhythms which diminish with further brain maturation (Boord et al., 2007; Clarke et al., 2001; Dustman et al., 1999; Gasser et al., 1988; Gibbs and Knott, 1949; John et al., 1980; Matousek and Petersen, 1973; Wackermann and Matousek, 1998). This effect also extends to sleep (Campbell and Feinberg, 2009; Feinberg and Campbell, 2010), and has been suspected to mirror the development of higher cognitive functions (Case, 1992; Thatcher, 1994), and deviations from normal oscillatory patterns has been associated with lagged or abnormal brain maturation (John et al., 1980). That these developmental amplitude reductions are frequency specific, and are also found with magnetoencephalography (MEG) which is insensitive to changes in physical properties such as bone conductivity (Puligheddu et al., 2005; Takeshita et al., 2002), corroborates their neurodevelopmental nature. Again, very few studies specifically focused on late maturational changes (Whitford et al., 2007) although many studies investigated (or included) EEG changes during childhood, when most dramatic changes occur. Typically, linear or curvilinear regression analyses are used to assess developmental effects from childhood to adulthood. Overall age-related changes are well captured using such models but the drawback is a lack of sensitivity to changes during specific, short periods. In particular, (post-)adolescent changes are rarely treated as a maturational step of its own and thus tend to be obscured by stronger developmental effects in childhood. For example, several scientists (Boord et al., 2007; Gasser et al., 1988; Whitford et al., 2007) found low-frequency EEG amplitude reduction associated with late maturation, but the curvilinear developmental regression effects appear to be driven mainly by the younger subjects. The specific contribution of the presumably more subtle changes due to late maturation remains unclear unless studied in a smaller age range confined to the specific (post-)adolescent transition from adolescence to adulthood using regression or age group contrasts. Whitford et al. suggested gray matter loss or synaptic pruning as a candidate 
to explain developmental EEG amplitude reduction (Whitford et al., 2007). Despite these structural changes that parallel the changes in EEG, the question remains whether there are also functional changes related to the EEG amplitude reduction with increasing age.

In recent years, simultaneous EEG and fMRI has allowed to link electrophysiological scalp-recorded activity more directly to cortical and subcortical regions. The exact physiological relationship between EEG amplitudes and the fMRI blood oxygen-level dependency (BOLD) signal remains unclear (Laufs, 2008). However, the two signals covary during rest in terms of their temporal fluctuation (following appropriate convolution to account for the lag and the lower frequency range of the BOLD signal), indicating that they are functionally coupled. Accordingly, the term coupling as used here does not describe the physiological mechanism underlying their coupling, but the correlation reflecting the statistical similarity between their (convolved) time courses. Research groups have repeatedly verified a thalamocortical circuit associated with alpha (8 - 13 Hz) oscillations (de Munck et al., 2007; Difrancesco et al., 2008; Feige et al., 2005; Goldman et al., 2002; Goncalves et al., 2006; Moosmann et al., 2003; Tyvaert et al., 2008). Furthermore, Scheeringa and colleagues found the so-called default mode network (Raichle et al., 2001; Raichle and Snyder, 2007) to be (inversely) correlated with frontal midline theta power (Scheeringa et al., 2008). Other authors found that different EEG rhythms reflect different functional networks (Laufs et al., 2006; Laufs et al., 2003b; Mantini et al., 2007). A consistent finding of concurrent EEG-fMRI studies is that at least for the lower frequencies, EEG power is inversely related to the BOLD signal, indicating that these EEG signals become stronger with decreased neuronal activity in associated brain regions. To our knowledge, research on resting state using simultaneous EEG-fMRI has so far been limited to healthy adults. The coupling of EEG rhythms to the BOLD signal has not been studied in developmental or clinical settings. Furthermore, little is known about the coupling during eyes-open resting state. Although eyes-open/eyes-closed protocols were used in prior EEG-fMRI resting state studies (Ben-Simon et al., 2008; Feige et al., 2005; Henning et al., 2006; Yang et al., 2009) (mainly to induce alpha modulation), none of these studies analyzed the eyes-open condition separately except for the work by Scheeringa et al. (Scheeringa et al., 2008) addressing eyes-open-theta coupling. Also, most of the previous studies exploring the EEG-BOLD coupling during rest were limited to specific components of the EEG such as eyes-closed occipital alpha or eyes-open frontal midline theta power, using selected electrode sites or ICA components. A comprehensive study of resting state EEG-BOLD coupling covering the common spectral frequency bands and resting states has not yet been performed. Such a study is important, because the profile formed by different EEG rhythms is characteristic of different brain states and brain functions, and because eyes-open and eyes-closed states differ substantially in levels of arousal and EEG (Barry et al., 2007; Berger, 1929 ; Bianciardi et al., 2009; Marx et al., 2004; Marx et al., 2003) as well as in fMRI (Bianciardi et al., 2009; Marx et al., 2004; Marx et al., 2003).

The aim of this study was to investigate late maturation of the brain's resting state using simultaneous EEG-fMRI. In particular, we asked whether changes in EEG-BOLD 
coupling parallel developmental EEG amplitude decreases. To investigate this question we compare EEG, fMRI and EEG-BOLD coupling differences between adults and adolescents. According to the literature we expected that adolescents have stronger EEG activity, especially in lower frequencies, although at this age the effect was expected to be subtle. We hypothesized that such increased EEG activity would reflect a distinct immature resting state network, and show coupling to different brain regions, or differ in coupling strength, rather than a constant coupling as expected for simple amplitude scaling of the EEG- or BOLD time series. Although differences were mostly expected in lower frequency bands, we included faster oscillations for comparison. Both eyes-open and eyes-closed conditions were included to provide a comprehensive picture of EEG-BOLD coupling and its late development during common resting states.

\section{Methods}

\section{Patricipants}

Two sex matched groups participated in this study. The 18 adults (mean age $24.9 \pm$ 3.8 years, 8 males) were recruited by university billboard ads and 18 adolescents (mean age $15.4 \pm 1.1$ years, 8 males) were recruited in school classes by approaching their teachers. All participants met the MRI safety standards, were healthy with no history of medical or psychiatric disease, and had normal or corrected to normal vision. All were right-hand dominant as assessed by the Edinburgh Handedness Inventory (Oldfield, 1971). All participants, as well as the children's parents, gave written informed consent prior to the investigation. The study was approved by the local ethics committee and was conducted in accordance with the guidelines determined in the Helsinki Declaration.

\section{Procedure}

After EEG preparation, participants were introduced to the nature of EEG signals and artifacts produced by movement and muscle tension. EEG and fMRI was recorded simultaneously in two 10 min resting state sessions with a 16 min working memory test (not reported here) in between. Each session consisted of alternating eyes-open (EO) and eyes-closed (EC) blocks of 2.5 min duration. One session started with eyes-open (EO-EC-EO-EC) and one with eyes-closed (EC-EO-EC-EO). The order of the two sessions was counterbalanced across participants by group and sex. A video beamer outside the scanner room projected onto a screen placed in front of the MR table. A mirror mounted on the head coil enabled participants to see through the bore onto the screen. In the eyes-open blocks a fixation star was presented on the screen, and at the end an instruction was given to close the eyes. The instruction to open the eyes after an eyes-closed block was given by a somatosensory stimulus on the left index finger via a pneumatic device. Short stimulation pulses were given already prior to scanning, to prevent subjects from any knee-jerk movements. Participants were instructed to follow the eyes-open/eyes-closed instruction, relax and refrain from any movements. For increased comfort, participants 
were fitted with a custom made felt cushion filling the space between the electrodes at the back of the head. The head was immobilized using foam pads and participants were provided with earplugs. Participants were personally checked after each scan session for electrode heating, tiredness or any indisposition.

\section{Data acquisition}

The EEG was recorded inside the scanner using $5 \mathrm{kHz}$ sampling rate, $32 \mathrm{mV}$ input range, $0.1-250 \mathrm{~Hz}$ bandpass filters, and data transmission from two MR-compatible BrainAmp amplifiers (Brain Products, Gilching, Germany) via optic fibers outside the scanner room. Sixty scalp electrodes were recorded using MR-compatible caps (easycap, Munich, Germany) with twisted and fixed electrode cables in three different editions to account for different head sizes of the participants. All electrode positions of the 10-20 system plus the following 10-10 system sites were used: $\mathrm{FPz}, \mathrm{AFz} \mathrm{FCz}, \mathrm{CPz}, \mathrm{POz}, \mathrm{Oz}$, Iz, F5/6, FC1/2/3/4/5/6, FT7/8/9/10, C1/2/5/6, CP1/2/3/4/5/6, TP7/8/9/10, P5/6, PO1 $/ 2 / 9 / 10, \mathrm{OI} 1 / 2)$. O $1 / 2$ and $\mathrm{FP} 1 / 2$ were placed $2 \mathrm{~cm}$ laterally from the standard positions for more even coverage (Brem et al., 2010; Maurer et al., 2007). F1 served as recording reference, F2 was the ground electrode. Two additional electrodes were placed below the outer canthus of each eye and two further electrodes right to the sternum and on the left chest close to the heart to record the electrocardiogram (ECG). Electrode impedances were kept below $20 \mathrm{k} \Omega$. The EEG was monitored while scanning using online correction software (RecView Brain Products, Gilching, Germany) and checked for signal quality and eyes-open/close transitions. FMRI data were acquired on a 3.0 T (GE Healthcare, Milwaukee, WI, USA) whole-body scanner using a standard head coil. Prior to fMRI acquisition a shimming procedure was used to minimize susceptibility distortions due to local static magnetic field inhomogeneities. Functional MR data were acquired with $\mathrm{T} 2{ }^{*}$-sensitive multi-slice echo planar imaging (EPI) sequence (TR $=1.815 \mathrm{~s} ; \mathrm{TE}=32 \mathrm{~ms} ; \mathrm{FOV}=22 \mathrm{~cm}$; image matrix $=64 \times 64$; voxel size $=3.44 \mathrm{x}$ $3.44 \times 3.8 \mathrm{~mm}^{3}$; flip angle $=75^{\circ}, 33$ axial slices covering the whole brain). One session consisted of 336 volumes. The first 6 volumes were excluded to avoid magnetic saturation effects.

\section{EEG processing and analysis}

Brain Vision Analyzer software (version 1.05, Brain Products, Gilching, Germany) served for offline processing of the EEG. MR-gradient artifacts were removed by average artifact subtraction (Allen et al., 2000; Allen et al., 1998) as implemented in the software (sliding average over $50 \mathrm{TRs}$, down sampled to $500 \mathrm{~Hz}$ and lowpass filtered at $70 \mathrm{~Hz}$ cutoff). To eliminate the ballistocardiogram artifact (BCG) a very similar subtraction procedure was used, with artifact window aligned on QRS complexes detected in ECG traces, templates based on 10 consecutive pulse intervals, and individually estimated time delay for subtraction based on global field power (GFP) distribution (CBC Parameters, Version 1.1). Subsequently, ECG channels were discarded from further analysis (QRS markers were kept). Data was digitally bandpass filtered $(0.5-70 \mathrm{~Hz}, 24 \mathrm{~dB} /$ oct and $50 \mathrm{~Hz}$ 

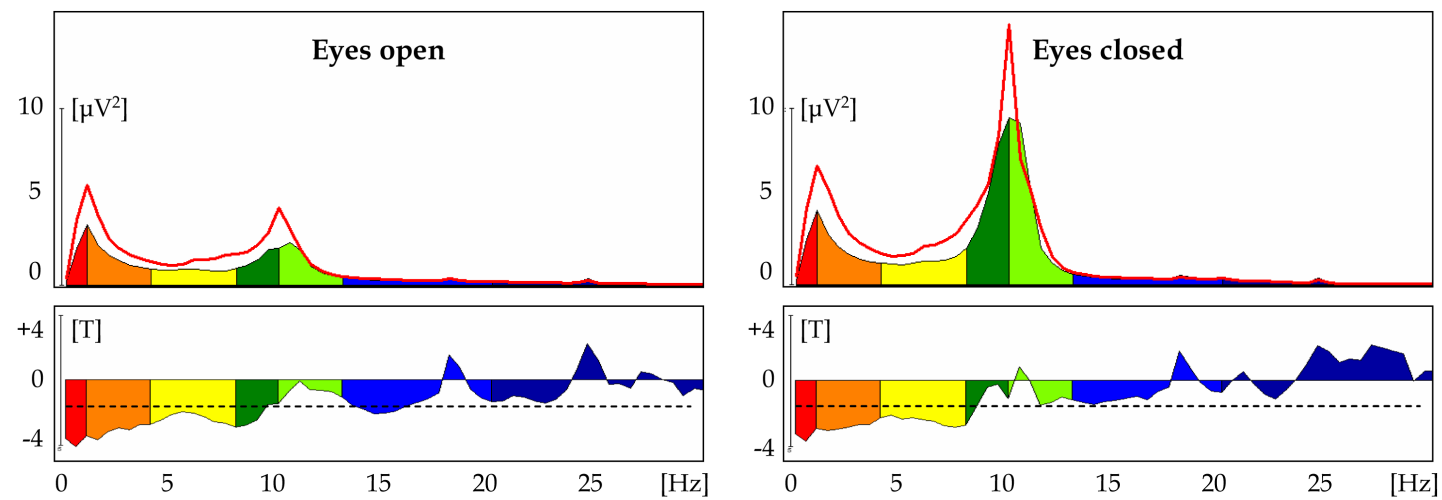

Fig. 1.1 Adolescence is characterized by increased low frequency EEG amplitudes. Group mean of global spectral power (GSP) for adults (colored spectra) and adolescents (red line) separate for eyes-open (left) and eyes-closed (right) condition. Lower panels: t-values of the group contrast. Dashed line: level of significance $(\mathrm{t}(34)=1.69, \mathrm{p}<0.05$, one-tailed, uncorrected for multiple comparison) .

Notch) and downsampled to $256 \mathrm{~Hz}$. An infomax ICA (Delorme and Makeig, 2004) was calculated on the concatenated data sets of the two sessions. Movement-related artifacts which may not be suitable for ICA correction were excluded from the unmixing procedure. Components were profiled by their topography, activation time course, spectrogram and contribution to averaged BCG amplitude. Components clearly assigned to either eye blinks (Jung et al., 2000), residual gradient artifacts or residual BCG artifacts were excluded from the back projection. The EEG was then transformed to the average reference (Lehmann and Skrandies, 1980) and segments with remaining artifacts were marked and excluded from successive analysis.

EEG regressor construction: First the EEG of each participant was split into the two separate sessions to evaluate possible session differences, and further divided into eyes-open and eyes-closed condition. The condition onsets were defined individually by the exact time of opening and closing the eyes, as indicated by ICA components activation traces. The data were parsed into contiguous epochs triggered by the scan starts, resulting in 330 time points per session. The data were further parsed into epochs of the length of a TR. Absolute spectral power was estimated for each epoch using Fast Fourier Transformation (FFT, Hanning window: $10 \%$, zero padded, resolution 0.5 $\mathrm{Hz}$ ). To estimate the total activity over the scalp, global spectral power (GSP) was calculated for each frequency point as the root mean square across all FFT-transformed scalp channels (Jann et al., 2009; Michels et al., 2010). The mean spectral band value was calculated for delta $(1-3 \mathrm{~Hz})$, theta $(4-7.5 \mathrm{~Hz})$, alpha1 $(8-10 \mathrm{~Hz})$, alpha2 $(10-13$ $\mathrm{Hz})$, beta1 $(14-19 \mathrm{~Hz})$ and beta2 $(20-30 \mathrm{~Hz})$. GSP estimates for artifactual segments (see above) were linearly interpolated. The GSP time series were normalized and thus recruited for fMRI model specification (see below). Additional regional EEG regressors were built based on subsets of electrodes in order to account for conventional locally defined EEG rhythms. For the delta and theta band frontal electrodes AFz, Fz, F3/4 and $\mathrm{FCz}$ were used. For alpha1 and alpha2 occipital electrodes $\mathrm{O} 1 / 2$, and for alpha2 and beta1 central electrodes $\mathrm{C} 3 / 4$ were used. 
EEG-BOLD Correlations During (Post-)Adolescent Brain Maturation

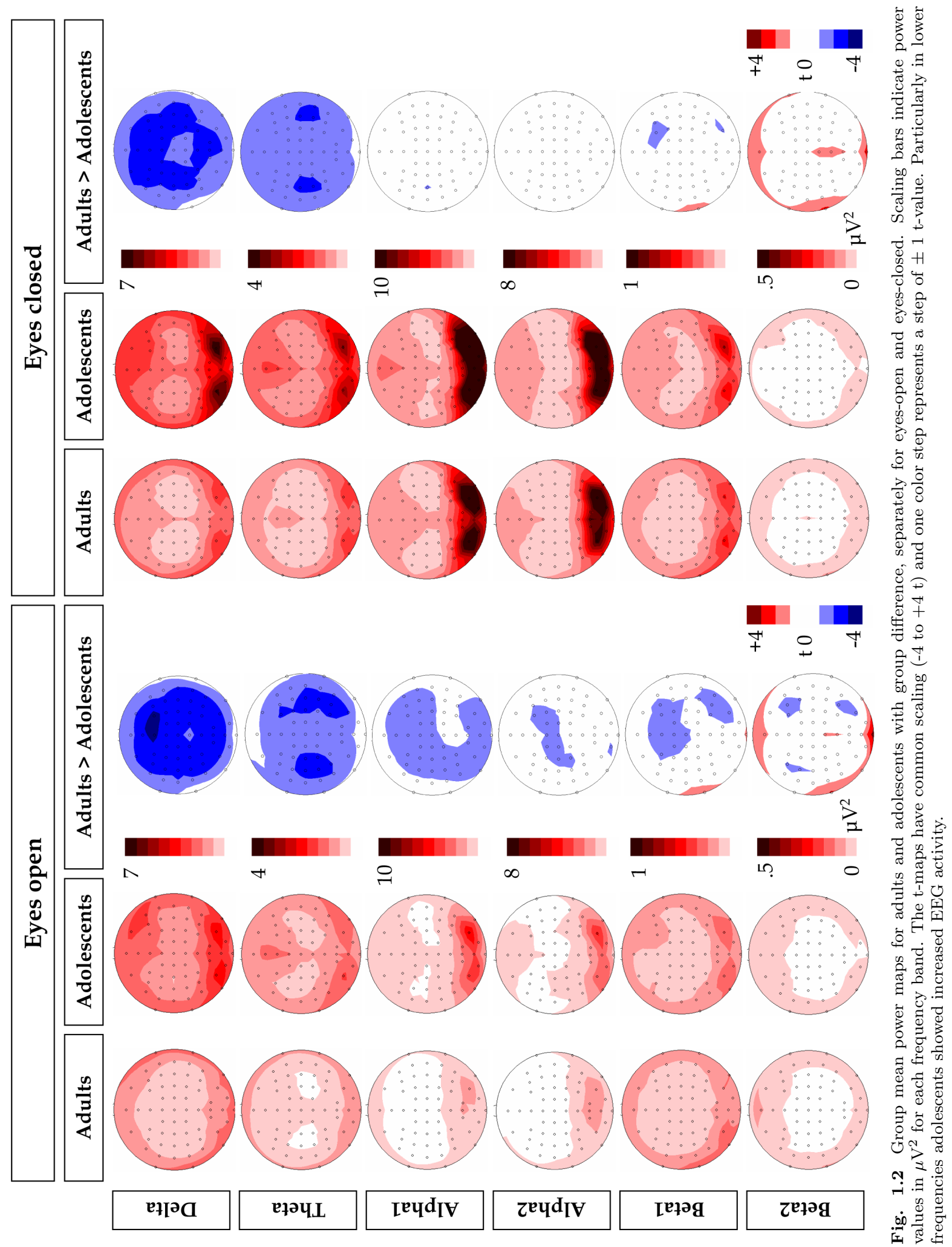


The EEG was analyzed in terms of GSP as well as electrode-wise to conserve topographic information. For each condition separately, spectral power values were averaged across FFT epochs and averaged within the above frequency bands. Group means were compared using t-statistics.

\section{FMRI processing and analysis}

Preprocessing and statistical analysis of fMRI data was done with SPM5 (Wellcome Department of Cognitive Neurology, London, UK). The two sessions were preprocessed separately. Images were realigned to the first scan to correct for head motion, and motion parameters were later included in the statistical model. No participant needed to be excluded due to extensive head motions (i.e. motion $>2 \mathrm{~mm}$ or $>2^{\circ}$ ). To prepare for group analysis, images were normalized to the Montreal Neurological Institute (MNI) standard brain, resampled to isotropic $3 \mathrm{~mm}^{3}$ voxels and smoothed with a Gaussian kernel (isotropic FWHM of $9 \mathrm{~mm}$ ). A standard HRF was used for convolution of model regressors. To account for serial correlations, an autoregressive model of the first order was used. Highpass filtering with standard $128 \mathrm{~s}$ cutoff eliminated slow signal drifts. Both sessions were analyzed separately to test for session differences. If session differences were not of interest, individual contrast images were calculated from session average. In any case session specific motion parameters were modeled as confounds.

The fMRI analysis modeled BOLD fluctuation, explained by EEG power fluctuations, using a general linear model (GLM). Eyes-open and eyes-closed GSP regressors of each frequency band (see EEG processing above) were used as parametric modulation of the eyes-open and eyes-closed blocks. The transitions between eyes-open and eyes-closed blocks were modeled in separate regressors of no interest, since motor activity and strong visual input is seen here as confounding both EEG and fMRI resting-state signals. In the main analysis, each frequency band was analyzed in a separate model. In a second analysis, focusing only on frequency-specific coupling, all frequency bands were entered into one common GLM. Separate models reveal the complete correlation pattern of a frequency whereas a common model reveals the partial correlation pattern of a frequency at the single subject level. By definition, the latter is only a part of the former. A disadvantage of the common model is that it is blind to the common variance among all regressors (i.e., their covariance) that will not be attributed to any of the partial collinear regressors (e.g. general power increase by eyes-closing, stimulation or even during rest). The common model may increase specificity (Tyvaert et al., 2008) and may therefore be of much interest, but separate models remain essential to detect less frequency-specific effects. Individual contrast images were calculated for eyes-open and eyes-closed GSP modulation regressors and used for random effects analysis. For the main analysis as well as for the common GLM analysis, a $2 \times 2 \times 6$ analysis of variance (ANOVA) with factors group (adults, adolescents), condition (eyes-open, eyes-closed) and frequency (delta, theta, alpha1, alpha2, beta1, beta2) was applied to calculate group statistics. Because the ANOVA revealed strong main effects of condition and frequency of secondary interest (see Results section), EEG-BOLD coupling patterns were reported as post-hoc t-tests (in both directions). Since only condition and frequency specific t-contrasts allow 


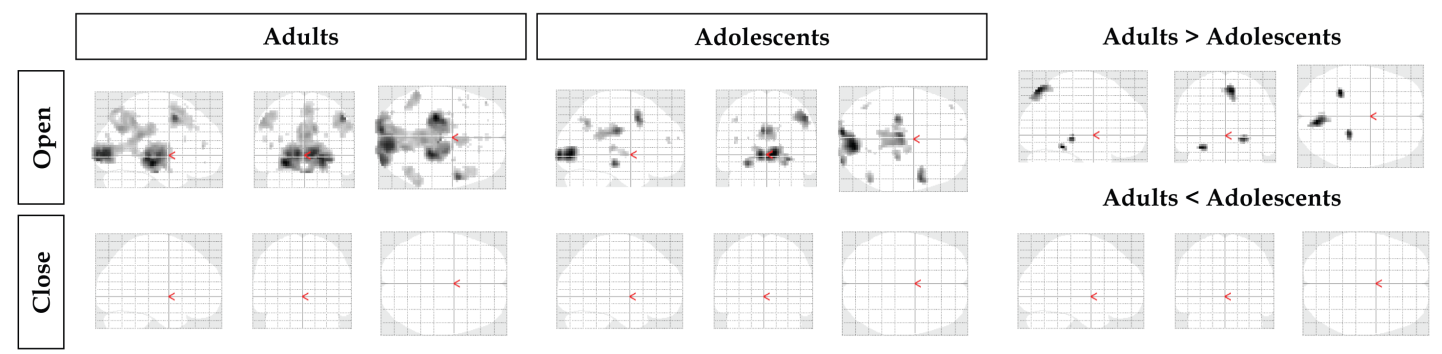

Fig. 1.3 Conventional fMRI analysis without EEG regressors. Group means of individual 'eyes-open > eyesclosed' ("open") and 'eyes-open < eyes-closed' ("close") contrasts are shown at p < 0.05 FWE corrected. Group difference thresholded at $\mathrm{p}<0.001$ uncorrected, corrected with minimal cluster size of 25 voxels.

connect to the existing literature on EEG-BOLD coupling, these ANOVA results are attached in supplementary Fig. 1. 1. The three regional EEG regressors (frontal delta and theta, occipital alpha1 and alpha2, central alpha2 and beta1) were calculated in separate random effects analysis.

Conventional fMRI analysis aimed to analyze eyes-open and eyes-closed condition independent from EEG. The model was identical to the one described above except no EEG modulation was included. This model was calculated using a $128 \mathrm{~s}$ highpass filter, and in a post-hoc analysis also with a $600 \mathrm{~s}$ filter. The first model is analogous to the EEG-informed models, whereas the second uses the adequate filter length to retain also the very slow eyes-open/eyes-closed paradigm cycles of $600 \mathrm{~s}$. Random statistics were calculated based on the individual eyes-open versus eyes-closed contrast image.

Group mean statistics are reported at the $\mathrm{p}<0.05$ significance level, controlled for the family wise error rate (FWE) to account for the problem of multiple comparisons. For the purpose of interpretation only clusters contain at least 20 significant voxels are reported. The second analysis using a common model for all frequencies reached less statistical power and was reported at $\mathrm{p}<0.001$ corrected for multiple comparisons by minimal cluster extent of 25 voxels (Forman et al., 1995; Slotnick et al., 2003). Because group differences did not reach significance at the a priori threshold, results are shown at an uncorrected voxel-threshold of $\mathrm{p}<0.001$ (explanation in results and in discussion). Regions of interest (ROIs) were functionally defined from these group differences to plot local group mean contrast values.

\section{Results}

We did not identify significant differences between the two sessions, neither for EEG, fMRI, nor EEG-fMRI coupling data. Thus we report on data pooled from both recording sessions. 


\section{EEG results}

Results from both groups replicated the typical resting state EEG characterized by a sharp alpha peak (around $10 \mathrm{~Hz}$ ), with occipital topography, which was reduced in strength with eyes open. T-tests between the two age groups revealed higher power values for adolescents in lower frequencies $(<10 \mathrm{~Hz}$ ), as hypothesized (Fig. 1.1 and 1.2). Higher frequencies did not substantially distinguish between the two groups, and the difference even tended to invert. This held for GSP (Fig. 1.1) as well as for the power maps (Fig. 1.2), while the effect was equivalent in both eyes-open and eyes-closed condition.

\section{FMRI results}

Conventional fMRI results during eyes-open indicated that the thalamus, visual cortex and frontal eye fields were activated (Fig. 1.3). The adolescent group showed very similar activation within the visual system. During eyes-closed no regions of increased activation were found. Comparing the two groups, some regions were more activated in adults but none were more activated in adolescents. Interestingly, recalculating this analysis using a $600 \mathrm{~s}$ highpass filter to accommodate also very slow sustained BOLD effects turned out to yield very similar activations.

\section{EEG-BOLD correlation results}

The ANOVA results (supplementary Fig. 1.1) indicated strong and wide-spread main effects of condition and frequency. Therefore t-contrasts by frequency and condition are reported to specify these global effects and relate them to the existing literature on EEG-BOLD coupling. The main effect of group was small compared to the effects of the other factors. The group differences were restricted to visual areas peaking at $[18,-84,15]$ xyz MNI-coordinate. This difference arose mainly from the eyes-open beta1 band as clarified by t-contrasts with a local maximum in the same region (Fig. 1.4) and region of interest (ROI) analysis (Fig. 1.6), both described below. Interactions did not reach significance at the a priori statistical threshold (except for a few isolated voxels).

Patterns of EEG-BOLD coupling differed between frequencies, conditions and groups (Fig. 1.4 and Fig. 1.5). In all frequency bands, negative correlations were more prominent than positive ones. During eyes-open, more frequency bands showed BOLD correlates than during eyes-closed (Table 1.1).

During eyes-open delta band power correlated negatively with the BOLD signal in the adolescent group in the right inferior parietal lobe. Theta power was negatively correlated with left inferior parietal lobe BOLD in both groups. In adults, theta power and BOLD was also coupled in the right inferior parietal lobe and superior and middle frontal gyrus, both of which are commonly associated with the DMN (see also Fig. 1.6). Both alpha bands are associated to similar brain regions in occipital, parietal and frontal lobes. Beta1 power revealed a very distinct coupling pattern in both groups 
in parietal and frontal cortices commonly associated as attention network (Fox et al., 2005).

During eyes-closed the alpha2 band was linked to a thalamocortical pattern in adults and adolescents. Group differences were not significant, but for adolescents alone, the positive correlation with the thalamus did not survive the strict statistical threshold. This correlation is displayed in more detail in Fig. 1.6. The beta1 and alpha2 bands elicited similar coupling, as the thalamus was again positively correlated in adults and the broad negative correlations were already present in adolescents.

The direct group comparison using a two-sample t-test did not reveal any group differences at the a priori threshold. When lowering the statistical threshold, differences first appeared in eyes-open alpha 2 and beta bands and eyes-closed beta2 at $\mathrm{p}<$ 0.001 uncorrected for multiple comparisons, as shown in Fig. 1.4 and 2.5. For these effects, the directions of the EEG-BOLD correlation of both groups are shown in Fig. 1.7 .

EEG-BOLD correlations with all frequencies in one common model are shown in supplementary Fig. 1.2 and 2.3. In the ANOVA, the main effects were significant only for factor frequency. Group by frequency interaction reached significance in a few left temporal and right parietal/superior temporal and lateral prefrontal clusters. Condition and frequency interacted in the thalamus, along the precentral and medial frontal gyri, in the parahippocampal gyrus and in the precuneus. Compared to separate GLMs, the common model produced a frequency main effect that was similarly distributed but more diffuse. It eliminated the main effects of group and condition as well as the group by condition interaction, but yielded more group $\mathrm{x}$ frequency interactions and differently distributed condition by frequency interactions.

Post-hoc t-tests of the common model correlations yielded only few correlations if corrected by FWE method and were displayed at a more lenient threshold (supplementary Fig. 1.3). Low frequencies delta and theta showed partially similar patterns in both conditions, including positively correlated occipital cortex/precuneus, pre- and postcentral gyri, anterior cingulate cortex and temporal and frontal lobe regions along the Sylvian fissure and negatively correlated clusters in DMN-associated areas including medial prefrontal cortex, posterior cingulated cortex and inferior parietal lobe. During eyes-open predominantly negative correlations were found in occipital cortex/precuneus for alpha2 and in attention-control-network-related areas in lateral parietal/frontal lobes in beta1. During eyes-closed, positive thalamic correlations reached significance in beta1 in adults and adolescents and in beta 2 in adults. Negative eyes-closed correlations appeared in alpha2, beta1 and beta2 in occipital, parietal, temporal or pre-/postcentral regions, together reflecting the formerly established thalamocortical pattern. All three higher frequencies accounted partially for this pattern but the frequency specificity appeared not robust when comparing the two groups. Compared to separate GLMs, the common model demonstrated less overall statistical power. The low frequency delta and theta negative DMN association did not change between the two analyses. In contrast, broad positive correlations appeared in these frequencies in regions similar to the negative correlated part of the thalamocortical pattern. This low frequency pattern was specific to both delta 
Results

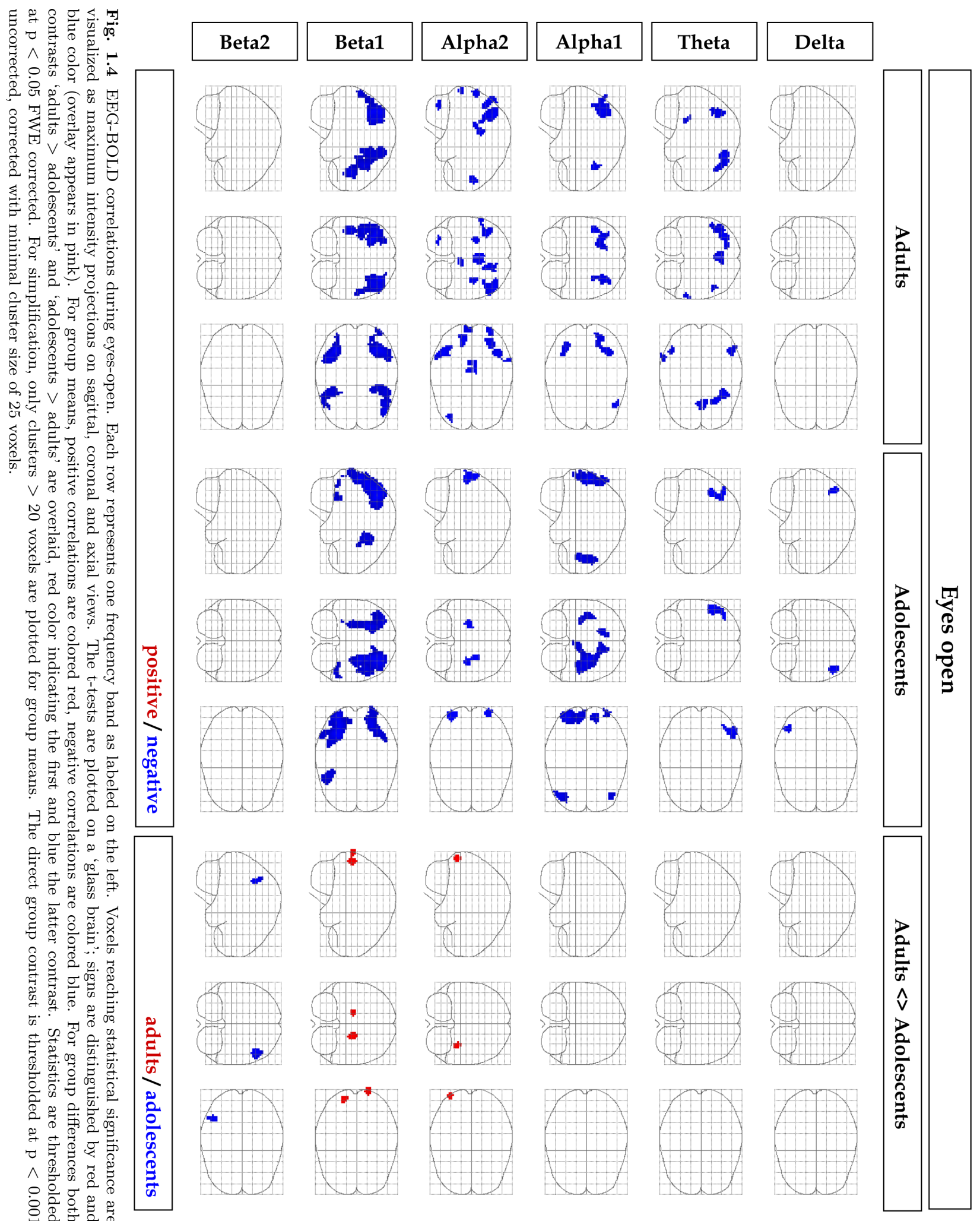




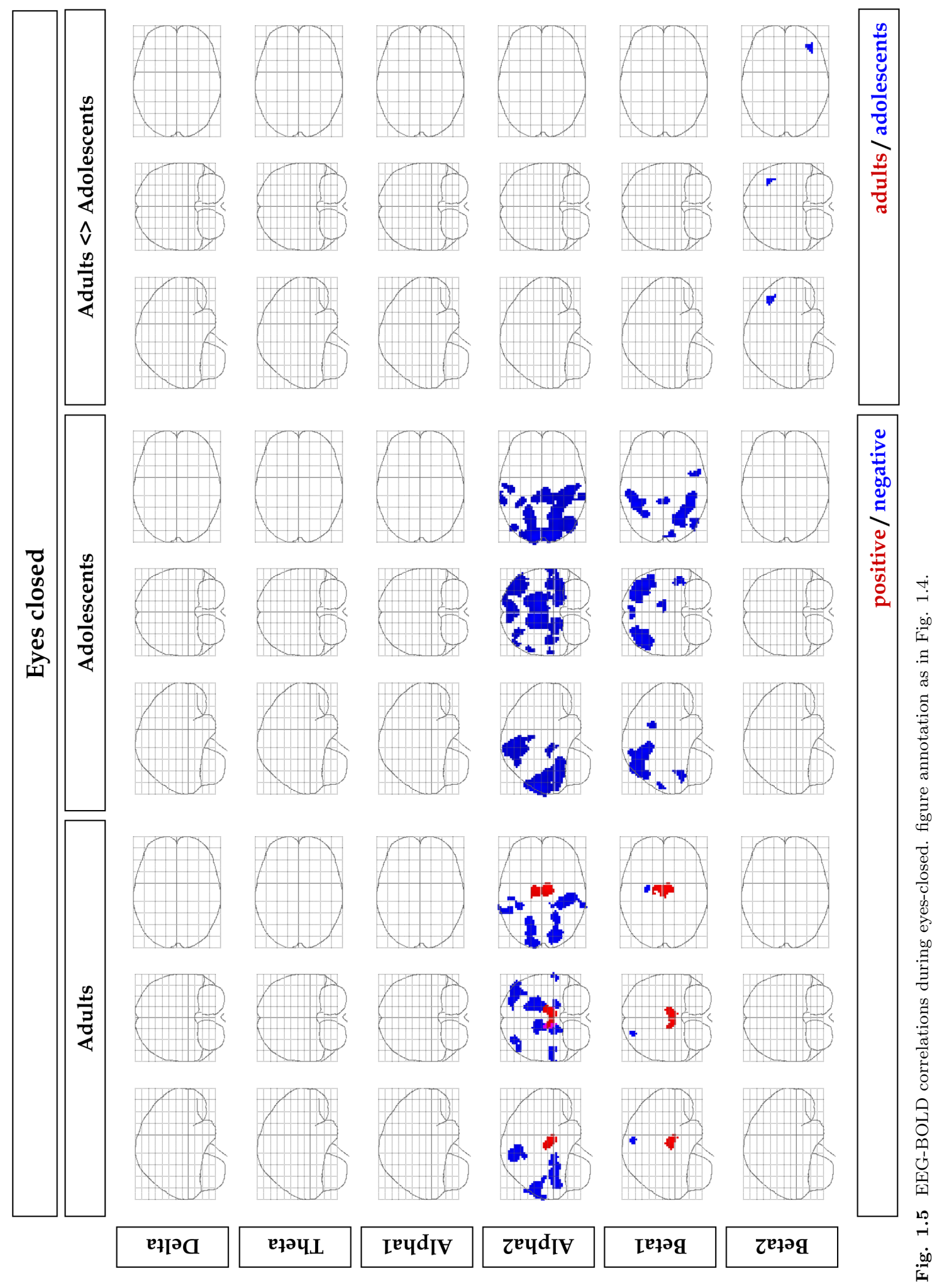


and theta bands, appeared quite robust between adults and adolescents and sustained the alternating conditions. The former redundancy between eyes-open alpha1, alpha2 and beta1 was eliminated in the common model attributing occipital correlations specifically to alpha2 and the attention-control network correlations specifically to beta1. Compared to separate GLMs, the eyes-closed thalamocortical pattern was split up the between all three upper frequency bands by the partial correlation approach. Group differences did not reach significance in this analysis, except for a small cluster in eyes-open alpha1 in right cingulated at $[18,-45,24]$ peak xyz MNI-coordinate and eyes-closed delta in right inferior parietal lobe at $[57,-54,36]$ that did not spatially overlap with the correlation pattern of the corresponding frequency.

\section{Discussion}

Characteristic maturational EEG amplitude reduction of low-frequency oscillations were found after age 15, although this effect was less prominent in absolute terms than the decreases reported during infancy, childhood and early adolescence. Unlike studies focusing on adolescents of a similar mean age but including younger, pre-adolescent participants driving the decrease (Gasser et al., 1988; Whitford et al., 2007), we directly compared groups of older adolescents and young adults. Although, based on the literature, only subtle spectral EEG differences could be expected between these groups, we still found substantial reduction of low-frequency EEG amplitudes at this late stage of brain maturation. Despite this EEG age effect, both groups revealed the typical resting state EEG characteristics of strong posterior alpha oscillations with eyes-closed. For theta, a frontal component could also be distinguished. The developmental low frequency power reductions tended to be similar across scalp regions (Fig. 1.2., see also Whitford et al. 2007). Therefore the global GSP measure seems to appropriately summarize (post-)adolescent EEG maturation in one comprehensive measure (Fig. 1.1).

The conventional fMRI results (independent of EEG activity) revealed no such age related group differences (Fig. 1.3). During eyes-open, the visual pathway including thalamus, geniculate laterale nuclei and visual cortex together with frontal eye fields were activated in both groups. These activations can be expected since open eyes produce a stimulation of the visual system which is not the case if the eyes are closed. Furthermore the result is in line with other fMRI studies contrasting eyes-open and eyes-closed states (Marx et al., 2004; Marx et al., 2003). The eyes-closed condition did not elicit any regions of greater activation. Although the standard highpass filter eliminated sustained paradigm induced variance, the reanalysis using less filtering (not shown) did not affect the results. Hence BOLD signal fluctuations responsible for the condition difference mainly consist of faster fluctuations than those sustained over the full eyes-open or eyes-closed periods (300 s each). While the fMRI indicated increased activity during eyes-open, the EEG activity in contrast (in particular within alpha band, consistent with the classical observation of Berger, 1929) was increased during eyes-closed. This result is in line with other observations that synchronization of background EEG is often correlated with states of lower arousal or suppression of brain areas (Barry et al., 2007; 
EEG-BOLD Correlations During (Post-)Adolescent Brain Maturation

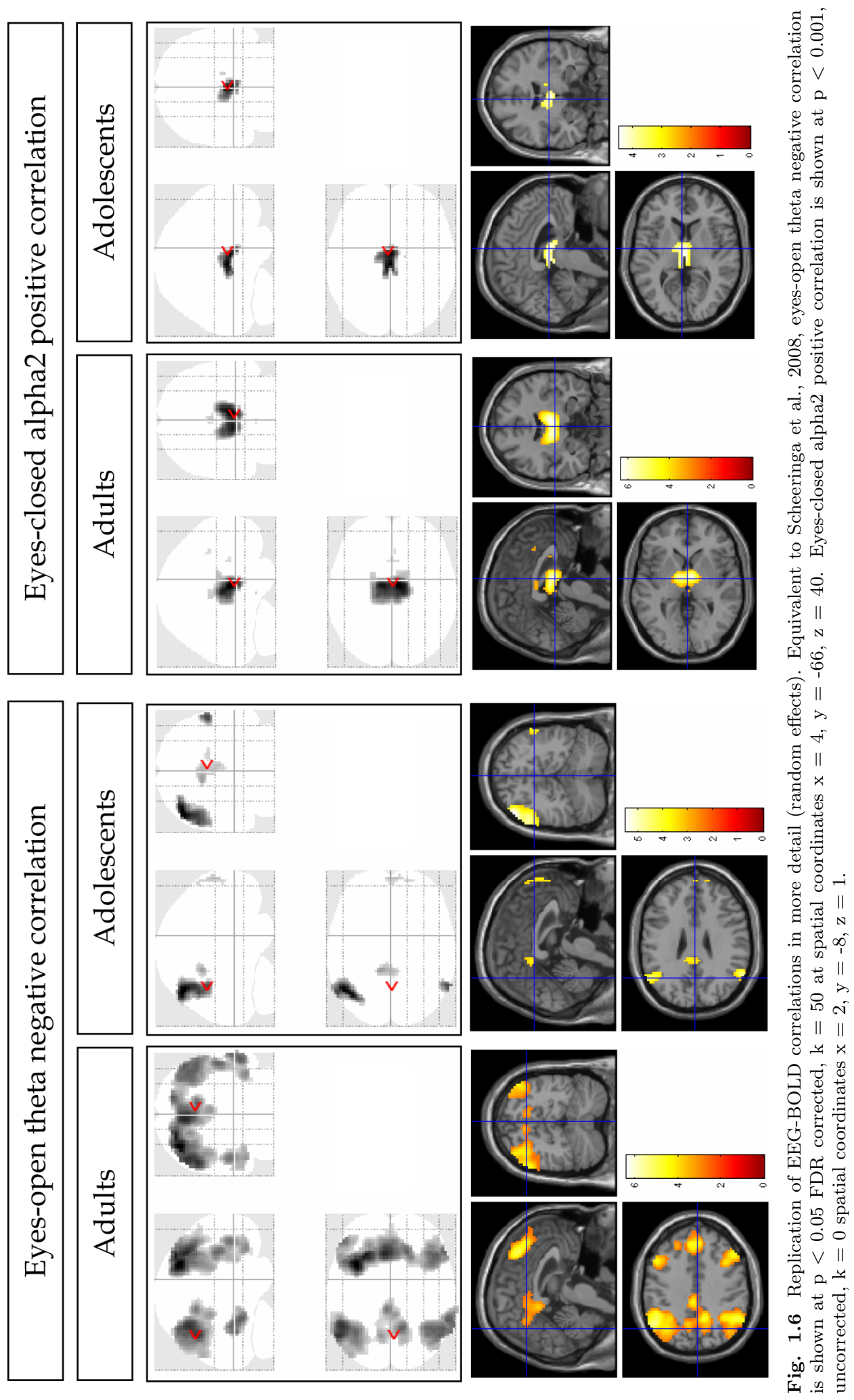


Pfurtscheller, 2001), and illustrates the inverse relationship between lower frequency EEG and fMRI.

The EEG-BOLD correlation results of adults correspond well to the existing literature. Many core findings of previous studies were found replicated: (i) EEG power correlations with the BOLD signal were predominantly negative (de Munck et al., 2007; Feige et al., 2005; Goldman et al., 2002; Laufs et al., 2003a; Moosmann et al., 2003; Ritter et al., 2009; Tyvaert et al., 2008). This inverse relationship is in contrast to studies that find local field potentials (LFP) positively associated with the BOLD signal (Logothetis et al., 2001; Nir et al., 2007; Viswanathan and Freeman, 2007). A possible explanation is that in contrast to LFP at least some EEG rhythms reflect a local deactivation of brain functioning through phasing neuron populations (Pfurtscheller, 2001). (ii) During eyes-open the DMN was anticorrelated with theta power. The adult pattern found here overlaps with previous work in several regions, including right middle temporal gyri, bilaterally inferior parietal lobule and bilaterally inferior frontal gyri (Scheeringa et al., 2008). Scheeringa and colleagues used a spatially constrained theta power represented by a single ICA component. In contrast GSP as used in this study includes signals from all scalp sites without weighting by statistical independence (Jann et al., 2009; Michels et al., 2010). Accordingly our coupling pattern can be interpreted as the global features of theta EEG power. This correlation is displayed in more detail in Fig. 1.6. (iii) During eyes-closed alpha power was negatively correlated in occipital and parietal cortices and positively correlated with the thalamus (de Munck et al., 2007; Difrancesco et al., 2008; Feige et al., 2005; Goldman et al., 2002; Goncalves et al., 2006; Moosmann et al., 2003; Tyvaert et al., 2008). It has been suggested that posterior alpha power represents down-regulation of the primary visual system during eyes-closed with the thalamus partly driving this process (de Munck et al., 2007; Feige et al., 2005; Goldman et al., 2002). However some alpha-BOLD correlation studies could not reveal a positive correlation of the thalamus (Jann et al., 2009; Laufs et al., 2003a). This inconsistency may be explained by the typical large variability of the EEG between individuals (Goncalves et al., 2006). The correlation pattern in our data extended from occipital to pericentral regions, and the thalamocortical pattern extended to the beta1 band. Positive thalamic BOLD correlation in beta band was reported before (de Munck et al., 2009; Moosmann et al., 2003). One reason for this robust pattern may be the inclusion of "rolandic" alpha (Gastaut, 1952; Salmelin and Hari, 1994) and beta (Pfurtscheller, 1981; Salmelin and Hari, 1994) rhythms in GSP. Similar to visual-related posterior alpha, these rhythms which relate to the thalamocortical regulation of motor and somatosensory systems were also found inversely correlated to the BOLD signal (Ritter et al., 2009). During eyes-closed, alpha2 and beta1 were the only frequencies correlated with the BOLD signal. To also account for specific, well defined local EEG rhythms (frontal midline theta, occipital alpha, central alpha and beta), separate analyses were carried out, based on regional sets of electrodes (supplementary Fig. 1.4 and 1.5). The correlation pattern from these local rhythms overlapped to a high degree with the GSP correlation pattern, but tended to be weaker than the latter.

Including both common variants of the resting state paradigm - eyes-open and eyes- 


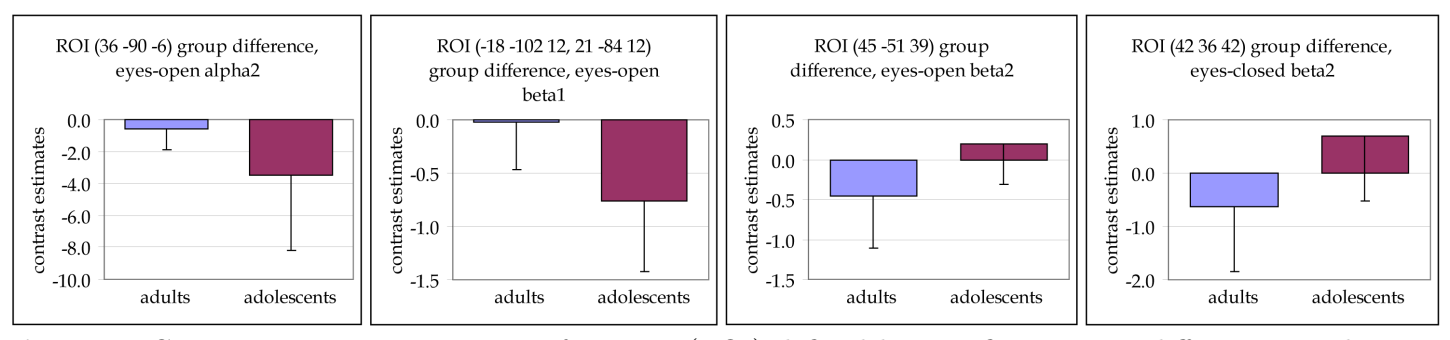

Fig. $\quad 1.7$ Contrast estimates in regions of interest (ROI) defined by significant group differences as shown in Fig. 1.4 and Fig. 1.5 for eyes-open alpha2, beta1, beta2 and eyes-closed beta2. The ROI's center is given in xyz-coordinates in the panel titles. In the first two panels adolescents have stronger negative EEG-BOLD coupling than adults. In the latter two panels, adolescents have positive but adults have negative coupling.

closed - as well as all major EEG frequency bands, we were able to draw a more comprehensive picture of resting state EEG-BOLD coupling than reported so far. This is important because the two resting conditions do differ substantially in both EEG (Barry et al., 2007; Berger, 1929 ) and fMRI (Bianciardi et al., 2009; Marx et al., 2004; Marx et al., 2003). Indeed our results were highly condition and partly frequency specific. Interestingly, the eyes-open condition - although somewhat neglected by researchers so far - elicited even more robust EEG-BOLD coupling patterns than the eyes-closed condition. During eyes-open the two alpha bands elicited similar correlations in parietal and frontal lobes. In contrast, during eyes-closed, splitting the alpha band elicited strikingly different results. A conspicuous pattern during eyes-open in the beta1 frequency range was robustly found in both groups. Beta1 GSP correlated negatively in extended bilateral parietal and frontal lobe regions forming the attention network (Fox et al., 2005). Overall we found four main EEG-BOLD coupling patterns in our eyes-open/eyes-closed resting state paradigm, corresponding to (i) the DMN; (ii) occipital cortex; (iii) the attention network; and (iv) a thalamocortical network. Our EEG-BOLD coupling pattern proved robust when based on random effects analysis corrected for multiple comparisons, allowing for reliable generalization to the population level. This contrasts with previous reports of EEG-BOLD coupling where a lack of significant results in random effect analyses was attributed to substantial inter-subject variability of EEG data (Goncalves et al., 2006; Laufs et al., 2006).

We also included an alternative EEG-BOLD coupling analysis to disentangle frequency specific effects. To this end we performed an additional common GLM analysis with all frequency bands entered simultaneously. This ANOVA based on the common model supported our conclusion regarding frequency specific coupling, as the main effect of frequency resembled the one obtained using separate GLMs. The common model analysis eliminated the main effects of group and condition. Consistent with its selective sensitivity to frequency specific effects, some of the condition effects in the separate models analysis appeared as condition by frequency interactions in the common model. The bandwise t-statistics revealed lower overall statistical power in the common model than in separate models. This probably reflects the loss of common variance across frequencies. The common model produced far more positive correlations, particularly in the low frequencies, which can be interpreted as relative rather than absolute increases in coupling. 
Further, the common GLM confirmed that the DMN is coupled to both, theta and delta independently, leading to the conclusion that DMN is coupled to low frequency EEG in general. This DMN-low frequency coupling appeared to be the most general EEG-fMRI resting state feature as it occurred independently of condition. The attention-control network related negative correlation during eyes-open was specific for the beta1 band. The common model produced some similarities with the separate models for the alpha and beta bands. However, the positive alpha-BOLD correlation in the thalamus during eyes closed - a landmark EEG-BOLD correlation - was eliminated by the common model. This suggests that the thalamic correlation was partly shared with other frequencies and therefore no longer attributed to alpha2 in the common GLM. Positive thalamic beta correlations were replicated in the common model, suggesting that thalamocortical beta coupling is more frequency specific. Positive thalamic BOLD correlation in beta band was reported before (de Munck et al., 2009; Moosmann et al., 2003). In the common GLM, all higher frequencies became partly sensitive to the negative correlation of the thalamocortical pattern but these correlations appeared not very robust across the two groups. Group differences of the common model analysis were even fewer and appeared in different frequencies than in the separate models analysis. Interestingly, eyes-closed delta was less negative coupled in adults than in adolescents in a small cluster associated with the negatively correlated DMN. This effect was model dependent as it was not evident in separate frequency correlations (next paragraph). Hence it is questionable if this small difference manifests a direct correspondence to the absolute power difference emerging in EEG maturation.

No study so far investigated EEG-BOLD coupling from a developmental perspective in general, or for late maturation in particular. Motivated by the late neurodevelopmental effect of decreasing slow oscillatory power, the primary goal was to investigate corresponding effects in the EEG-BOLD coupling. However, adults and adolescents did not differ in EEG-BOLD correlation in any frequency band. Accordingly, the mean coupling patterns of adults and adolescents overlapped to a high degree. Because of the centrality of this question, we explored trends for corresponding developmental differences of EEG-BOLD coupling using a lenient statistical thresholding $(\mathrm{p}<0.001$, uncorrected for multiple comparisons). Although these trends must be interpreted with caution, adolescents tended towards slightly stronger coupling of occipital regions with the alpha2 and beta1 bands (Fig. 1.4 and 1.5). This trend for stronger coupling parallels a more pronounced occipital EEG topography in these frequency bands in adolescents. However, these subthreshold effects emerged at higher frequencies, and therefore are likely to be unrelated to the significant power decrease during and after late adolescence in low frequencies. This observation held even if thresholded more liberally (i.e. $\mathrm{p}<0.005$ uncorrected, not shown), and even for the frequency specific supplementary analysis. Similarly none of the local EEG rhythms differed in their BOLD correlation pattern between adults and adolescents at the strict statistical threshold (supplementary Fig. 1.4 and 1.5). A minor trend emerged, but again not in the delta and theta bands, and thus not corresponding to the EEG maturation effects. Interestingly however, adolescents tended to have stronger (negative) correlation in post the central sulcus region for the 
Table. 1.1 Anatomical regions corresponding to the significant main results in Fig. 1.4 and Fig. 1.5. Listed are cluster size, coordinates in MNI space, peak t-value, corresponding anatomical region and Brodmann Area.

\begin{tabular}{|c|c|c|c|c|c|c|c|c|}
\hline & & & & & & & Adults & \\
\hline condition & frequency & contrast & \begin{tabular}{|l|}
$\begin{array}{l}\text { cluster } \\
\text { size }\end{array}$ \\
\end{tabular} & $\begin{array}{l}\mathrm{MNI} \\
\end{array}$ & $\begin{array}{l}\text { coordin } \\
x, y, y, z)\end{array}$ & & t-value anatomical structure & $\square_{B A}$ \\
\hline Eyes-open & Delta & negative & & & & & & \\
\hline & Theta & negative & 124 & -36 & 15 & 54 & 5.93 Left Superior Frontal Gyrus & BA 8 \\
\hline & & & 94 & 0 & 33 & 48 & 5.32 Left Superior Frontal Gyrus & BA 8 \\
\hline & & & 97 & -48 & -54 & 45 & 5.27 Left Inferior Parietal Lobule & BA 40 \\
\hline & & & 24 & 63 & -45 & -3 & 5.01 Right Middle Temporal Gyrus & BA 21 \\
\hline & & & 10 & 57 & 30 & 18 & 4.97 Right Inferior Frontal Gyrus & BA 46 \\
\hline & & & 11 & -48 & 42 & 3 & 4.83 Left Inferior Frontal Gyrus & BA 46 \\
\hline & & & 20 & 48 & -54 & 39 & 4.77 Right Inferior Parietal Lobule & BA 40 \\
\hline & & & 5 & 45 & 18 & 51 & 4.56 Right Middle Frontal Gyrus & BA 8 \\
\hline & Alpha1 & negative & 41 & -48 & 33 & 30 & 5.69 Left Middle Frontal Gyrus & BA 46 \\
\hline & & & 18 & -48 & 45 & 6 & 5.36 Left Inferior Frontal Gyrus & \\
\hline & & & 144 & -33 & -54 & 42 & 5.32 Left Inferior Parietal Lobule & BA 40 \\
\hline & & & 144 & 33 & -63 & 45 & 5.21 Right Superior Parietal Lobule & BA 7 \\
\hline & & & 11 & -63 & -42 & 33 & 5.09 Left Inferior Parietal Lobule & BA 40 \\
\hline & & & 10 & 12 & .72 & 45 & 4.54 Right Precuneus & BA 7 \\
\hline & Alpha2 & negative & 171 & 45 & -51 & 54 & 5.55 Right Inferior Parietal Lobule & BA 40 \\
\hline & & & 85 & 15 & -72 & 42 & 5.34 Right Precuneus & BA 7 \\
\hline & & & 84 & -39 & -51 & 42 & 5.30 Left Inferior Parietal Lobule & BA 40 \\
\hline & & & 99 & 6 & -24 & 33 & 5.30 Right Cingulate Gyrus & BA 23 \\
\hline & & & 45 & -30 & -87 & 24 & 5.29 Left Cuneus & BA 19 \\
\hline & & & 40 & -27 & -66 & -39 & 5.22 Left Pyramis & \\
\hline & & & 37 & 36 & 57 & 21 & 5.15 Right Superior Frontal Gyrus & BA 10 \\
\hline & & & 14 & 6 & -36 & 48 & 4.96 Right Precuneus & BA 7 \\
\hline & & & 35 & 6 & -93 & 0 & 4.82 Right Cuneus & BA 17 \\
\hline & & & 5 & 6 & 15 & 69 & 4.77 Right Superior Frontal Gyrus & BA 6 \\
\hline & & & 5 & -24 & -27 & 0 & 4.76 Left Thalamus & \\
\hline & & & 8 & 30 & -87 & 27 & 4.64 Right Cuneus & BA 19 \\
\hline & Beta1 & negative & 485 & 36 & -57 & 57 & 6.80 Right Superior Parietal Lobule & BA 7 \\
\hline & & & 502 & -33 & -57 & 45 & 6.53 Left Inferior Parietal Lobule & BA 40 \\
\hline & & & 534 & -45 & 42 & 6 & 6.46 Left Middle Frontal Gyrus & BA 46 \\
\hline & & & 292 & 39 & 12 & 57 & 5.49 Right Middle Frontal Gyrus & BA 6 \\
\hline & & & 7 & 48 & 42 & -6 & 4.67 Right Middle Frontal Gyrus & BA 47 \\
\hline & Beta2 & negative & 44 & 48 & -51 & 39 & 4.67 Right Inferior Parietal Lobule & BA 40 \\
\hline & & & 83 & -6 & -33 & 33 & 4.51 Left Cingulate Gyrus & BA 31 \\
\hline & & & 28 & -48 & -57 & 45 & 4.37 Left Inferior Parietal Lobule & BA 40 \\
\hline Eyes-closed & Alpha2 & positive & 186 & 9 & -6 & 3 & 5.79 Right Thalamus & \\
\hline & & & & -9 & -6 & 3 & 5.23 Left Thalamus & \\
\hline & & & & -12 & -18 & 12 & 4.79 Left Thalamus & \\
\hline & Alpha2 & negative & 196 & -18 & .57 & 0 & 5.40 Left Lingual Gyrus & BA 18 \\
\hline & & & 220 & 21 & .78 & 24 & 5.39 Right Precuneus & BA 31 \\
\hline & & & 70 & -48 & -42 & 3 & 5.32 Left Middle Temporal Gyrus & BA 22 \\
\hline & & & 242 & 54 & -21 & 57 & 5.15 Right Postcentral Gyrus & $\mathrm{BA} 3$ \\
\hline & & & 63 & 21 & -51 & -3 & 5.09 Right Parahippocam pal Gyrus & BA 30 \\
\hline & & & 74 & -33 & -36 & 60 & 4.99 Left Postcentral G yrus & $\mathrm{BA} 3$ \\
\hline & & & 121 & -15 & .87 & 27 & 4.95 Left Cuneus & BA 19 \\
\hline & & & 22 & 66 & -36 & 0 & 4.80 Right Middle Temporal Gyrus & BA 21 \\
\hline & & & 13 & 9 & .21 & 54 & 4.69 Right Medial Frontal Gyrus & BA 6 \\
\hline & & & 16 & 60 & -12 & 39 & 4.67 Right Precentral Gyrus & BA 6 \\
\hline & & & 5 & 18 & -72 & -9 & 4.52 Right Lingual Gyrus & BA 18 \\
\hline & Beta1 & positive & 138 & 0 & -9 & 3 & 5.51 Left Thalam us & \\
\hline & $\overline{\text { Beta }}$ & negative & 24 & -24 & -6 & 66 & 5.13 Left Middle Frontal Gyrus & BA 6 \\
\hline
\end{tabular}

\begin{tabular}{|c|c|c|c|c|c|}
\hline \multicolumn{6}{|c|}{ Adolescents } \\
\hline $\begin{array}{l}\text { size } \\
\text { size }\end{array}$ & & 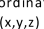 & & -value anatomical structure & BA \\
\hline 54 & 48 & -63 & 48 & 5.51 Right Inferior Parietal Lobule & BA 40 \\
\hline 263 & -48 & -57 & 36 & 5.35 Left Inferior Parietal Lobule & BA 40 \\
\hline 434 & 30 & -81 & 21 & 6.42 Right Middle Occipital Gyrus & BA 19 \\
\hline 150 & 48 & 48 & 12 & 5.64 Right Middle Frontal Gyrus & BA 10 \\
\hline 55 & -15 & -84 & 45 & 5.39 Left Precuneus & BA 7 \\
\hline 42 & -33 & -90 & 12 & 5.09 Left Middle Occipital Gyrus & BA 19 \\
\hline 55 & -45 & 45 & 21 & 5.02 Left Middle Frontal Gyrus & BA 46 \\
\hline 12 & 66 & -39 & 33 & 4.79 Right Inferior Parietal Lobule & BA 40 \\
\hline 7 & .24 & -81 & 21 & 4.64 Left Precuneus & BA 31 \\
\hline 5 & -6 & -99 & 6 & 4.53 Left Cuneus & BA 18 \\
\hline 203 & 33 & -81 & 12 & 5.00 Right Middle Occipital Gyrus & BA 19 \\
\hline 41 & -30 & -93 & 15 & 4.83 Left Middle Occipital Gyrus & BA 19 \\
\hline 14 & 3 & -93 & -3 & 4.76 Right Lingual Gyrus & BA 17 \\
\hline 988 & 33 & -51 & 51 & 6.66 Right Precuneus & BA 7 \\
\hline 577 & .24 & -69 & 36 & 6.03 Left Precuneus & BA 7 \\
\hline 195 & 48 & 15 & 33 & 5.41 Right Middle Frontal Gyrus & BA 9 \\
\hline 52 & 54 & -54 & -18 & 5.34 Right Inferior Temporal Gyrus & BA 20 \\
\hline 21 & 42 & -75 & -12 & 5.03 Right Inferior Occipital Gyrus & BA 19 \\
\hline 14 & -42 & -63 & -9 & 4.88 Left Middle Temporal Gyrus & BA 37 \\
\hline 9 & -18 & -90 & -6 & 4.75 Left Lingual Gyrus & BA 17 \\
\hline 16 & 45 & 51 & 3 & 4.71 Right Inferior Frontal Gyrus & BA 10 \\
\hline 6 & -51 & 27 & 30 & 4.68 Left Middle Frontal Gyrus & BA 46 \\
\hline 5 & 15 & -84 & -9 & 4.64 Right Lingual Gyrus & BA 18 \\
\hline
\end{tabular}

\begin{tabular}{|c|c|c|c|c|c|}
\hline 2218 & -9 & -93 & 33 & 6.79 Left Cuneus & BA 19 \\
\hline 873 & 45 & -18 & 60 & 5.74 Right Precentral Gyrus & BA 4 \\
\hline 43 & 66 & -27 & 6 & 5.15 Right Superior Temporal Gyrus & BA 22 \\
\hline 70 & -42 & -75 & 6 & 5.07 Left Middle Occipital Gyrus & BA 19 \\
\hline 68 & -30 & -36 & 54 & 5.07 Left Postcentral Gyrus & BA 3 \\
\hline 19 & 60 & & -12 & 4.95 Right Middle Temporal Gyrus & BA 21 \\
\hline 30 & -60 & -33 & 6 & 4.93 Left Superior Temporal Gyrus & BA 42 \\
\hline 32 & -45 & -18 & 63 & 4.87 Left Precentral Gyrus & BA 4 \\
\hline 6 & -36 & -27 & 69 & 4.79 Left Precentral Gyrus & BA 4 \\
\hline 25 & -57 & -12 & 39 & 4.76 Left Precentral Gyrus & BA 6 \\
\hline 431 & -42 & -42 & 54 & 5.77 Left Infer & BA 40 \\
\hline 543 & 27 & -60 & 63 & 5.67 Right Superior Parietal Lobule & BA 7 \\
\hline 74 & 48 & -66 & -6 & 5.10 Right Inferior Temporal Gyrus & BA 37 \\
\hline 48 & 57 & 9 & 36 & 4.90 Right Inferior Frontal Gyrus & BA 9 \\
\hline 52 & -6 & -36 & 66 & 4.86 Left Paracentral Lobule & BA 6 \\
\hline 12 & -21 & -75 & 45 & 4.81 Left Precuneus & BA 7 \\
\hline 37 & 12 & -84 & 24 & 4.74 Right Cuneus & BA \\
\hline & 30 & -3 & 72 & 4.73 Right Superior Frontal Gyrus & BA 6 \\
\hline
\end{tabular}

\begin{tabular}{|c|c|c|c|c|c|c|c|}
\hline condition & frequency & contrast & $\begin{array}{l}\begin{array}{l}\text { cluster } \\
\text { size }\end{array} \\
\end{array}$ & $\begin{array}{c}\text { MNI coord in } \\
(x, y, z)\end{array}$ & nate ${ }_{\text {t-value }}$ & anatomical structure & BA \\
\hline \multirow[t]{3}{*}{ Eyes-open } & Alpha1 & adults > adolescents & 30 & $\begin{array}{ll}36 & -90 \\
\end{array}$ & -6 & 72 Right Middle Occipital Gyrus & BA 18 \\
\hline & $\overline{\text { Beta1 }}$ & adults > adolescents & 40 & $\begin{array}{lr}21 & -84 \\
\end{array}$ & 12 & 3.8 Right Cuneus & BA 17 \\
\hline & $\overline{\text { Beta2 }}$ & adults > adolescents & 62 & $\begin{array}{ll}45 & -51\end{array}$ & 39 & 11 Right Inferior Parietal Lobule & BA 40 \\
\hline & & & & 42 & 42 & & A 9 \\
\hline
\end{tabular}


central beta1 rhythm (eyes-closed) than adults. We conclude that, in contrast to the developmental decrease of low frequency EEG activity, the frequency specific EEG-BOLD correlations do not change substantially at this late stage of brain maturation. Contrary to our initial hypothesis that a distinct network reflects late immaturity, but consistent with the simpler scaling hypothesis, the EEG-BOLD correlation pattern between the two signals remains largely the same despite changing magnitude of the low frequency EEG signal. This result indicates that the correlation pattern of EEG and fMRI signals mature earlier than the EEG amplitudes. In order to test whether the subthreshold coupling differences between adolescents and adults reflect genuine but asymptotic developmental effects, it will be necessary to contrast these data to data from younger children. However, the fact that the EEG-BOLD coupling does not mirror the neurodevelopmental process captured by the EEG, suggests that the two domains capture partly different aspects of brain functioning (Laufs, 2008).

The predominantly inverse relationships between EEG and fMRI signals in our data using the separate model may suggest that synchronized neuronal activity, at least for the lower frequency range up to the alpha band, is related to states of lower arousal, lower neural activity, and lower metabolic consumption: (i) EEG power fluctuations are negative correlated to the BOLD signal; (ii) EEG power is increased during eyes-closed whereas the BOLD signal is increased during eyes-open; (iii) EEG power mainly decreases with brain development, whereas the conventional fMRI analysis rather indicates an increasing trend with brain development.

\section{Conclusion}

In this study we investigated spectral EEG power changes between adolescence and adulthood for corresponding differences in the BOLD signal using simultaneously recorded fMRI during rest with eyes-open and eyes-closed periods. First, low-frequency EEG power continued to decrease beyond mid-adolescence. Second, similar robust EEG-BOLD coupling pattern was identified in adults and adolescents. Power in most EEG bands correlated negatively with the BOLD signal in areas of DMN and attention network during eyes-open, and in a thalamocortical network during eyes-closed. However, no robust changes in EEG-BOLD coupling corresponding to the decrease in low-frequency EEG power were identified. Our results indicate that the most prominent changes in EEG amplitudes are independent of the correlation with the BOLD signal during late maturation. To clarify at which developmental stage the mature pattern of EEG-BOLD coupling at rest emerges, future work needs to include younger participants.

\section{Acknowledgements}

This work was supported by the University Research Priority Program on Integrative Human Physiology. We like to thank Thomas Koenig for ICA implementation and Christian Gaser for some MATLAB code advice. 


\section{References}

Allen, P.J., Josephs, O., Turner, R., 2000. A method for removing imaging artifact from continuous EEG recorded during functional MRI. Neuroimage 12, 230-239.

Allen, P.J., Polizzi, G., Krakow, K., Fish, D.R., Lemieux, L., 1998. Identification of EEG events in the MR scanner: the problem of pulse artifact and a method for its subtraction. Neuroimage 8, 229-239.

Barry, R.J., Clarke, A.R., Johnstone, S.J., Magee, C.A., Rushby, J.A., 2007. EEG differences between eyes-closed and eyes-open resting conditions. Clin Neurophysiol 118, 2765-2773.

Beckmann, C.F., DeLuca, M., Devlin, J.T., Smith, S.M., 2005. Investigations into resting-state connectivity using independent component analysis. Philos Trans R Soc Lond B Biol Sci 360, 1001-1013.

Ben-Simon, E., Podlipsky, I., Arieli, A., Zhdanov, A., Hendler, T., 2008. Never resting brain: simultaneous representation of two alpha related processes in humans. PLoS One 3, e3984.

Berger, H., 1929 Über das Elektrenkephalogramm des Menschen. European Archives of Psychiatry and Clinical Neuroscience 87, 527-570.

Bianciardi, M., Fukunaga, M., van Gelderen, P., Horovitz, S.G., de Zwart, J.A., Duyn, J.H., 2009. Modulation of spontaneous fMRI activity in human visual cortex by behavioral state. Neuroimage 45, 160168.

Blakemore, S.J., Choudhury, S., 2006. Brain development during puberty: state of the science. Dev Sci 9 11-14.

Boord, P.R., Rennie, C.J., Williams, L.M., 2007. Integrating "brain" and "body" measures: correlations between EEG and metabolic changes over the human lifespan. J Integr Neurosci 6, 205-218.

Brem, S., Bach, S., Kucian, K., Guttorm, T.K., Martin, E., Lyytinen, H., Brandeis, D., Richardson, U., 2010. Brain sensitivity to print emerges when children learn letter-speech sound correspondences. Proc Natl Acad Sci U S A 107, 7939-7944.

Campbell, I.G., Feinberg, I., 2009. Longitudinal trajectories of non-rapid eye movement delta and theta EEG as indicators of adolescent brain maturation. Proc Natl Acad Sci U S A 106, 5177-5180.

Case, R., 1992. The role of the frontal lobes in the regulation of cognitive development. Brain Cogn 20, 5173 .

Clarke, A.R., Barry, R.J., McCarthy, R., Selikowitz, M., 2001. Age and sex effects in the EEG: development of the normal child. Clin Neurophysiol 112, 806-814.

Damoiseaux, J.S., Rombouts, S.A., Barkhof, F., Scheltens, P., Stam, C.J., Smith, S.M., Beckmann, C.F., 2006. Consistent resting-state networks across healthy subjects. Proc Natl Acad Sci U S A 103, 1384813853.

de Munck, J.C., Goncalves, S.I., Huijboom, L., Kuijer, J.P., Pouwels, P.J., Heethaar, R.M., Lopes da Silva, F.H., 2007. The hemodynamic response of the alpha rhythm: an EEG/fMRI study. Neuroimage 35, 11421151.

de Munck, J.C., Goncalves, S.I., Mammoliti, R., Heethaar, R.M., Lopes da Silva, F.H., 2009. Interactions between different EEG frequency bands and their effect on alpha-fMRI correlations. Neuroimage 47, 6976 .

Delorme, A., Makeig, S., 2004. EEGLAB: an open source toolbox for analysis of single-trial EEG dynamics including independent component analysis. J Neurosci Methods 134, 9-21.

Difrancesco, M.W., Holland, S.K., Szaflarski, J.P., 2008. Simultaneous EEG/functional magnetic resonance imaging at 4 Tesla: correlates of brain activity to spontaneous alpha rhythm during relaxation. J Clin Neurophysiol $25,255-264$

Dustman, R.E., Shearer, D.E., Emmerson, R.Y., 1999. Life-span changes in EEG spectral amplitude, amplitude variability and mean frequency. Clin Neurophysiol 110, 1399-1409.

Fair, D.A., Dosenbach, N.U., Church, J.A., Cohen, A.L., Brahmbhatt, S., Miezin, F.M., Barch, D.M., Raichle, M.E., Petersen, S.E., Schlaggar, B.L., 2007. Development of distinct control networks through segregation and integration. Proc Natl Acad Sci U S A 104, 13507-13512.

Feige, B., Scheffler, K., Esposito, F., Di Salle, F., Hennig, J., Seifritz, E., 2005. Cortical and subcortical correlates of electroencephalographic alpha rhythm modulation. J Neurophysiol 93, 2864-2872.

Feinberg, I., Campbell, I.G., 2010. Sleep EEG changes during adolescence: an index of a fundamental brain reorganization. Brain Cogn 72, 56-65.

Forman, S.D., Cohen, J.D., Fitzgerald, M., Eddy, W.F., Mintun, M.A., Noll, D.C., 1995. Improved assessment of significant activation in functional magnetic resonance imaging (fMRI): use of a cluster-size threshold. Magn Reson Med 33, 636-647.

Fox, M.D., Snyder, A.Z., Vincent, J.L., Corbetta, M., Van Essen, D.C., Raichle, M.E., 2005. The human brain is intrinsically organized into dynamic, anticorrelated functional networks. Proc Natl Acad Sci U S A 102, 9673-9678.

Gasser, T., Verleger, R., Bacher, P., Sroka, L., 1988. Development of the EEG of school-age children and adolescents. I. Analysis of band power. Electroencephalogr Clin Neurophysiol 69, 91-99. 
Gastaut, H., 1952. [Electrocorticographic study of the reactivity of rolandic rhythm.]. Rev Neurol (Paris) 87, 176-182.

Gibbs, F.A., Knott, J.R., 1949. Growth of the electrical activity of the cortex. Electroencephalogr Clin Neurophysiol 1, 223-229.

Giedd, J.N., Blumenthal, J., Jeffries, N.O., Castellanos, F.X., Liu, H., Zijdenbos, A., Paus, T., Evans, A.C., Rapoport, J.L., 1999. Brain development during childhood and adolescence: a longitudinal MRI study. Nat Neurosci 2, 861-863.

Gogtay, N., Giedd, J.N., Lusk, L., Hayashi, K.M., Greenstein, D., Vaituzis, A.C., Nugent, T.F., 3rd, Herman, D.H., Clasen, L.S., Toga, A.W., Rapoport, J.L., Thompson, P.M., 2004. Dynamic mapping of human cortical development during childhood through early adulthood. Proc Natl Acad Sci U S A 101, 81748179 .

Goldman, R.I., Stern, J.M., Engel, J., Jr., Cohen, M.S., 2002. Simultaneous EEG and fMRI of the alpha rhythm. Neuroreport 13, 2487-2492.

Goncalves, S.I., de Munck, J.C., Pouwels, P.J., Schoonhoven, R., Kuijer, J.P., Maurits, N.M., Hoogduin, J.M., Van Someren, E.J., Heethaar, R.M., Lopes da Silva, F.H., 2006. Correlating the alpha rhythm to BOLD using simultaneous EEG/fMRI: inter-subject variability. Neuroimage 30, 203-213.

Henning, S., Merboldt, K.D., Frahm, J., 2006. Task- and EEG-correlated analyses of BOLD MRI responses to eyes opening and closing. Brain Res 1073-1074, 359-364.

Jann, K., Dierks, T., Boesch, C., Kottlow, M., Strik, W., Koenig, T., 2009. BOLD correlates of EEG alpha phase-locking and the fMRI default mode network. Neuroimage 45, 903-916.

John, E.R., Ahn, H., Prichep, L., Trepetin, M., Brown, D., Kaye, H., 1980. Developmental equations for the electroencephalogram. Science 210, 1255-1258.

Jung, T.P., Makeig, S., Westerfield, M., Townsend, J., Courchesne, E., Sejnowski, T.J., 2000. Removal of eye activity artifacts from visual event-related potentials in normal and clinical subjects. Clin Neurophysiol 111, 1745-1758.

Laufs, H., 2008. Endogenous brain oscillations and related networks detected by surface EEG-combined fMRI. Hum Brain Mapp 29, 762-769.

Laufs, H., Holt, J.L., Elfont, R., Krams, M., Paul, J.S., Krakow, K., Kleinschmidt, A., 2006. Where the BOLD signal goes when alpha EEG leaves. Neuroimage 31, 1408-1418.

Laufs, H., Kleinschmidt, A., Beyerle, A., Eger, E., Salek-Haddadi, A., Preibisch, C., Krakow, K., 2003a. EEGcorrelated fMRI of human alpha activity. Neuroimage 19, 1463-1476.

Laufs, H., Krakow, K., Sterzer, P., Eger, E., Beyerle, A., Salek-Haddadi, A., Kleinschmidt, A., 2003b. Electroencephalographic signatures of attentional and cognitive default modes in spontaneous brain activity fluctuations at rest. Proc Natl Acad Sci U S A 100, 11053-11058.

Lehmann, D., Skrandies, W., 1980. Reference-free identification of components of checkerboard-evoked multichannel potential fields. Electroencephalogr Clin Neurophysiol 48, 609-621.

Logothetis, N.K., Pauls, J., Augath, M., Trinath, T., Oeltermann, A., 2001. Neurophysiological investigation of the basis of the fMRI signal. Nature 412, 150-157.

Luna, B., Padmanabhan, A., O'Hearn, K., 2010. What has fMRI told us about the development of cognitive control through adolescence? Brain Cogn 72, 101-113.

Mantini, D., Perrucci, M.G., Del Gratta, C., Romani, G.L., Corbetta, M., 2007. Electrophysiological signatures of resting state networks in the human brain. Proc Natl Acad Sci U S A 104, 13170-13175.

Marx, E., Deutschlander, A., Stephan, T., Dieterich, M., Wiesmann, M., Brandt, T., 2004. Eyes open and eyes closed as rest conditions: impact on brain activation patterns. Neuroimage 21, 1818-1824.

Marx, E., Stephan, T., Nolte, A., Deutschlander, A., Seelos, K.C., Dieterich, M., Brandt, T., 2003. Eye closure in darkness animates sensory systems. Neuroimage 19, 924-934.

Matousek, M., Petersen, I., 1973. Automatic evaluation of EEG background activity by means of age-dependent EEG quotients. Electroencephalogr Clin Neurophysiol 35, 603-612.

Maurer, U., Brem, S., Bucher, K., Kranz, F., Benz, R., Steinhausen, H.C., Brandeis, D., 2007. Impaired tuning of a fast occipito-temporal response for print in dyslexic children learning to read. Brain 130, 32003210 .

Michels, L., Bucher, K., Lüchinger, R., Klaver, P., Martin, E., Jeanmonod, D., Brandeis, D., 2010. Simultaneous EEG-fMRI during a working memory task: modulations in low and high frequency bands. PLoS One 5, e10298.

Moosmann, M., Ritter, P., Krastel, I., Brink, A., Thees, S., Blankenburg, F., Taskin, B., Obrig, H., Villringer, A., 2003. Correlates of alpha rhythm in functional magnetic resonance imaging and near infrared spectroscopy. Neuroimage 20, 145-158.

Nir, Y., Fisch, L., Mukamel, R., Gelbard-Sagiv, H., Arieli, A., Fried, I., Malach, R., 2007. Coupling between neuronal firing rate, gamma LFP, and BOLD fMRI is related to interneuronal correlations. Curr Biol 17, 1275-1285.

Oldfield, R.C., 1971. The assessment and analysis of handedness: the Edinburgh inventory. Neuropsychologia 9, 97-113. 
Paus, T., 2005. Mapping brain maturation and cognitive development during adolescence. Trends Cogn Sci 9 60-68.

Paus, T., Keshavan, M., Giedd, J.N., 2008. Why do many psychiatric disorders emerge during adolescence? Nat Rev Neurosci 9, 947-957.

Pfurtscheller, G., 1981. Central beta rhythm during sensorimotor activities in man. Electroencephalogr Clin Neurophysiol 51, 253-264.

Pfurtscheller, G., 2001. Functional brain imaging based on ERD/ERS. Vision Res 41, 1257-1260.

Puligheddu, M., de Munck, J.C., Stam, C.J., Verbunt, J., de Jongh, A., van Dijk, B.W., Marrosu, F., 2005. Age distribution of MEG spontaneous theta activity in healthy subjects. Brain Topogr 17, 165-175.

Raichle, M.E., MacLeod, A.M., Snyder, A.Z., Powers, W.J., Gusnard, D.A., Shulman, G.L., 2001. A default mode of brain function. Proc Natl Acad Sci U S A 98, 676-682.

Raichle, M.E., Snyder, A.Z., 2007. A default mode of brain function: a brief history of an evolving idea. Neuroimage 37, 1083-1090; discussion 1097-1089.

Ritter, P., Moosmann, M., Villringer, A., 2009. Rolandic alpha and beta EEG rhythms' strengths are inversely related to fMRI-BOLD signal in primary somatosensory and motor cortex. Hum Brain Mapp 30, 1168-1187.

Salmelin, R., Hari, R., 1994. Characterization of spontaneous MEG rhythms in healthy adults. Electroencephalogr Clin Neurophysiol 91, 237-248.

Scheeringa, R., Bastiaansen, M.C., Petersson, K.M., Oostenveld, R., Norris, D.G., Hagoort, P., 2008. Frontal theta EEG activity correlates negatively with the default mode network in resting state. Int J Psychophysiol $67,242-251$.

Sisk, C.L., Foster, D.L., 2004. The neural basis of puberty and adolescence. Nat Neurosci 7, 1040-1047.

Slotnick, S.D., Moo, L.R., Segal, J.B., Hart, J., Jr., 2003. Distinct prefrontal cortex activity associated with item memory and source memory for visual shapes. Brain Res Cogn Brain Res 17, 75-82.

Sowell, E.R., Thompson, P.M., Tessner, K.D., Toga, A.W., 2001. Mapping continued brain growth and gray matter density reduction in dorsal frontal cortex: Inverse relationships during postadolescent brain maturation. J Neurosci 21, 8819-8829.

Stevens, M.C., 2009. The developmental cognitive neuroscience of functional connectivity. Brain Cogn 70, 112 .

Takeshita, K., Nagamine, T., Thuy, D.H., Satow, T., Matsuhashi, M., Yamamoto, J., Takayama, M., Fujiwara, N., Shibasaki, H., 2002. Maturational change of parallel auditory processing in school-aged children revealed by simultaneous recording of magnetic and electric cortical responses. Clin Neurophysiol 113, 14701484.

Thatcher, R.W., 1994. Cyclic cortical reorganization: Origins of human cognitive development. In: Fischer, G.D.K.W. (Ed.), Human behavior and the developing brain. Guilford Press, New York, pp. 232-266.

Tyvaert, L., Hawco, C., Kobayashi, E., LeVan, P., Dubeau, F., Gotman, J., 2008. Different structures involved during ictal and interictal epileptic activity in malformations of cortical development: an EEG-fMRI study. Brain 131, 2042-2060.

van den Heuvel, M.P., Hulshoff Pol, H.E., 2010. Exploring the brain network: a review on resting-state fMRI functional connectivity. Eur Neuropsychopharmacol 20, 519-534.

Viswanathan, A., Freeman, R.D., 2007. Neurometabolic coupling in cerebral cortex reflects synaptic more than spiking activity. Nat Neurosci 10, 1308-1312.

Wackermann, J., Matousek, M., 1998. From the 'EEG age' to a rational scale of brain electric maturation. Electroencephalogr Clin Neurophysiol 107, 415-421.

Whitford, T.J., Rennie, C.J., Grieve, S.M., Clark, C.R., Gordon, E., Williams, L.M., $2007 . \quad$ Brain maturation in adolescence: concurrent changes in neuroanatomy and neurophysiology. Hum Brain Mapp 28, 228237.

Yang, L., Liu, Z., Rios, C., Yuan, H., He, B., 2009. Electrophysiological neuroimaging: cortical correlates of alpha rhythm modulation. Conf Proc IEEE Eng Med Biol Soc 2009, 1934-1936. 


\section{Supplementary material}

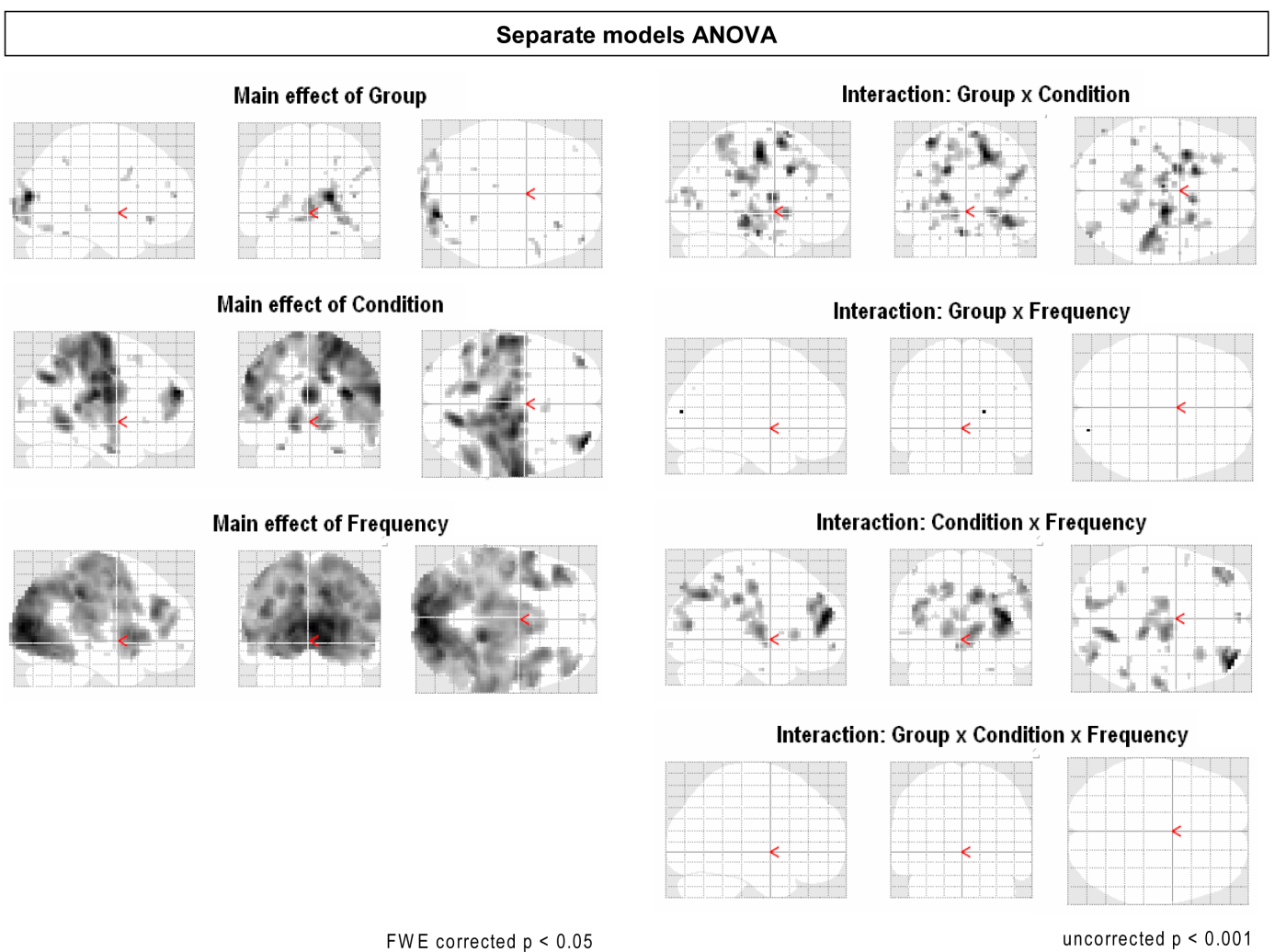

Suplementary Fig. 1.1 ANOVA results of the separate models analysis with main effects and interactions of the Group (adults, adolescents) x Condition (eyes-open, eyes-closed) x Frequency (delta, theta, alpha1, alpha2, beta1, beta2). Main effects are thresholded at $\mathrm{p}<0.05$, FWE corrected. Interactions did not survive FWE correction and are shown at $\mathrm{p}<0.001$, uncorrected $(\mathrm{k}=0)$. 
Common model ANOVA

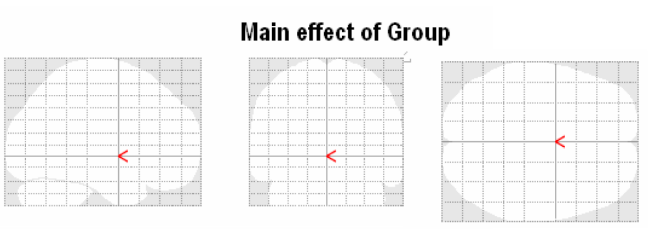

Main effect of Condition
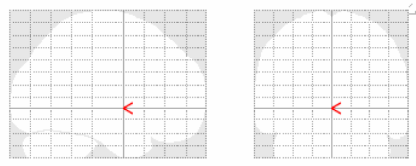

Main effect of Frequency
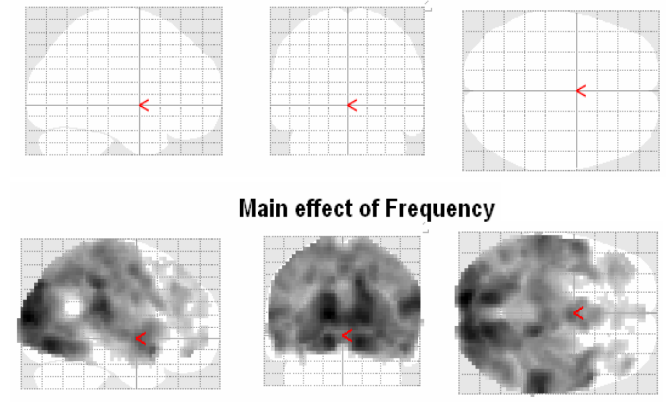

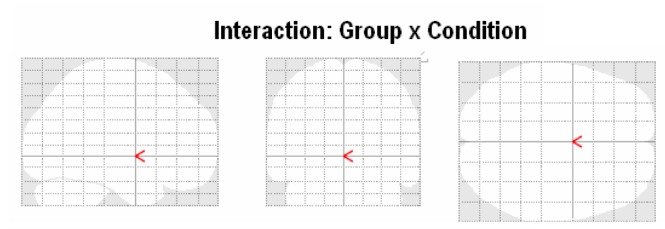

Interaction: Group x Frequency
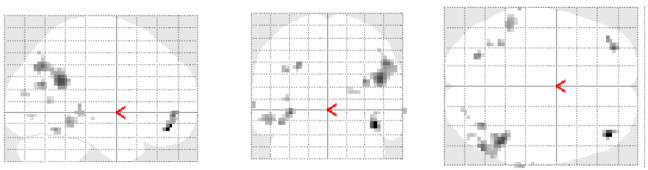

Interaction: Condition x Frequency

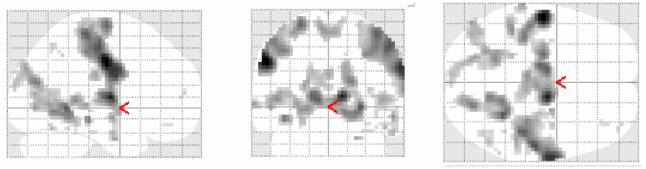

Interaction: Group x Condition x Frequency
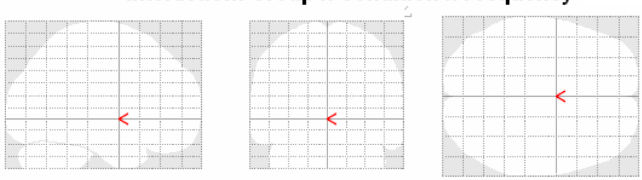

uncorrected $p<0.001$

Suplementary Fig. 1.2 ANOVA results for common model analysis with main effects and interactions of the Group (adults, adolescents) x Condition (eyes-open, eyes-closed) x Frequency (delta, theta, alpha1, alpha2, beta1, beta2). Main effects are thresholded at $\mathrm{p}<0.05$, FWE corrected. Interactions did not survive FWE correction and are shown at $\mathrm{p}<0.001$, uncorrected $(\mathrm{k}=0)$. 
Supplementary material

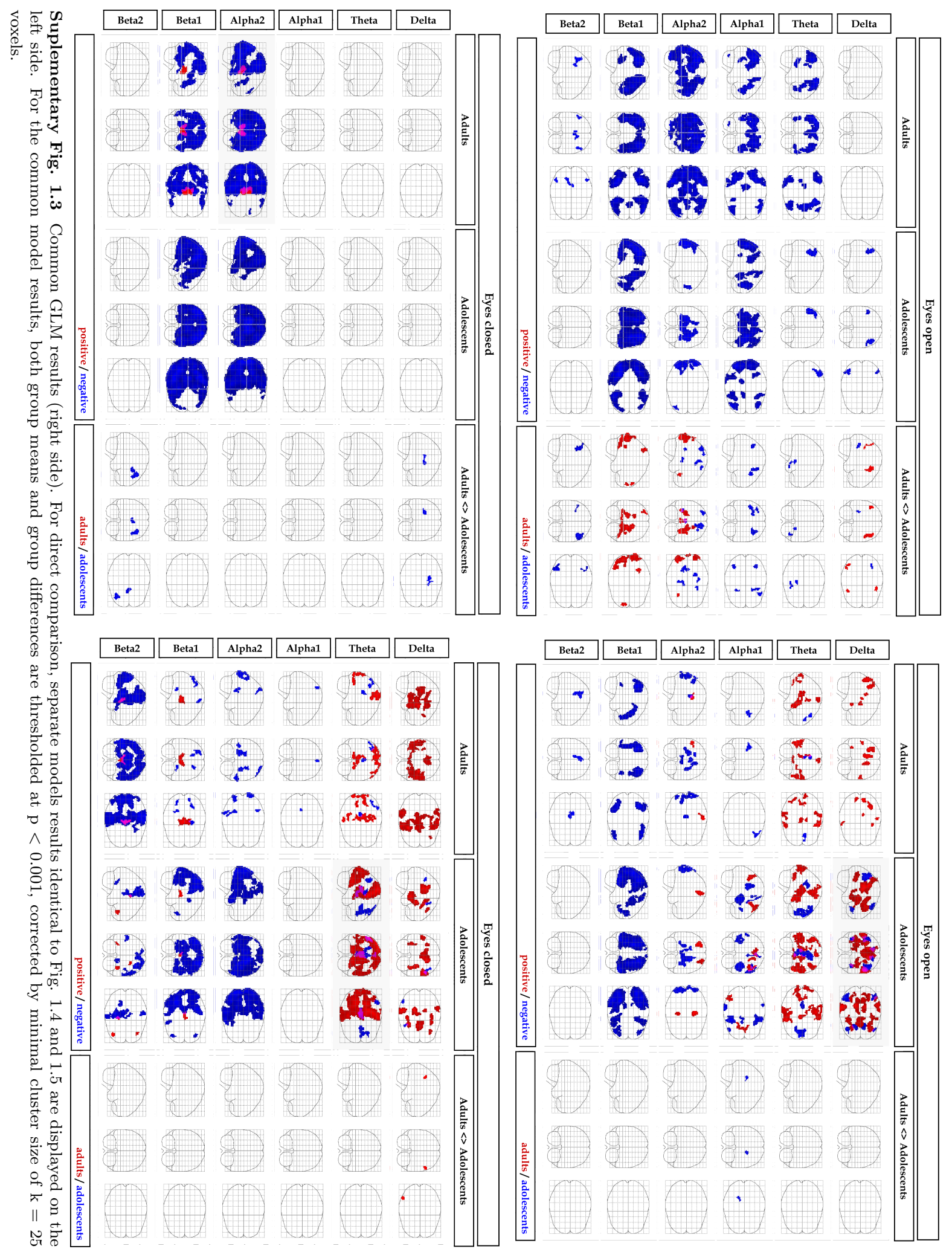




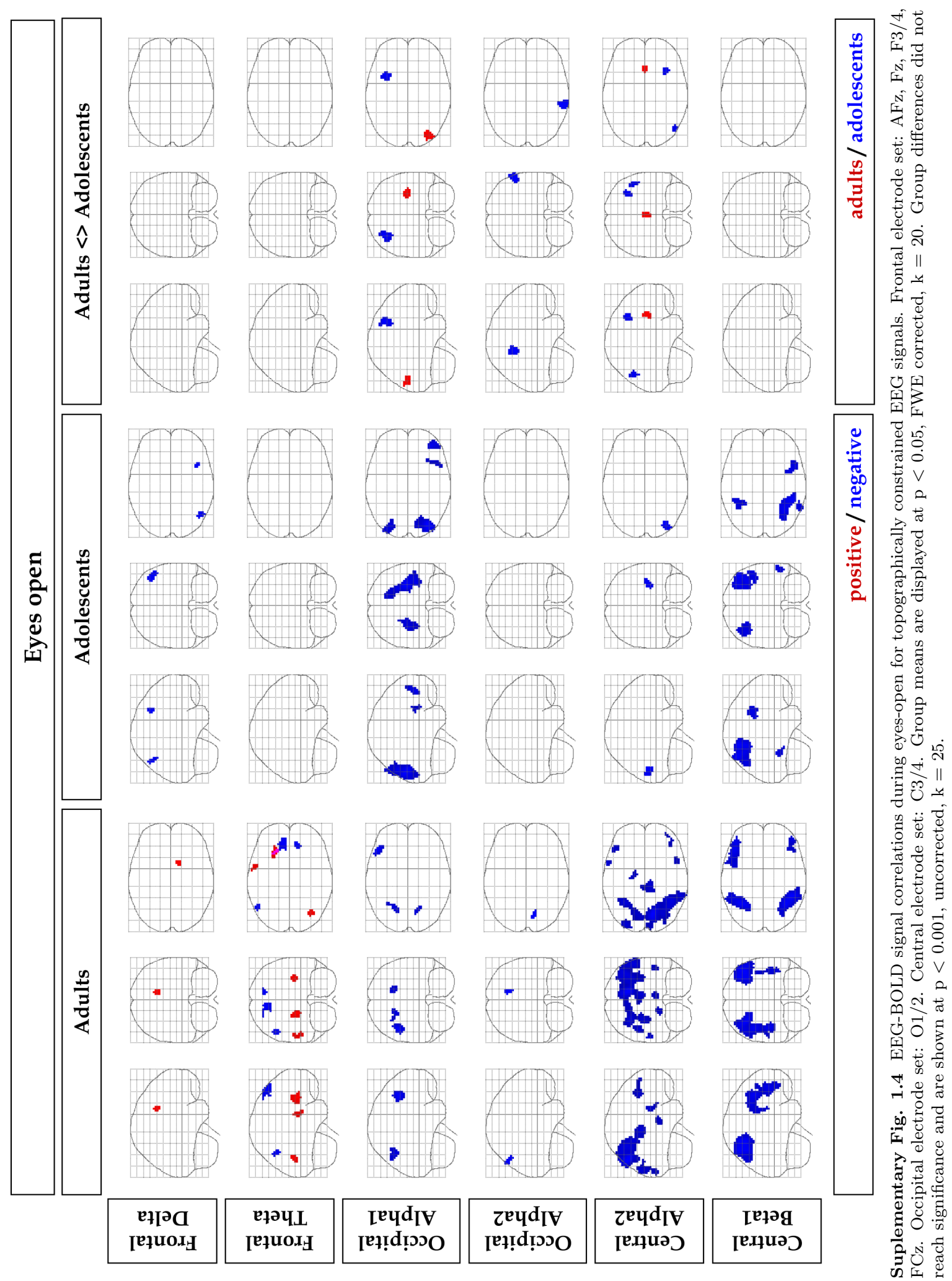


Supplementary material

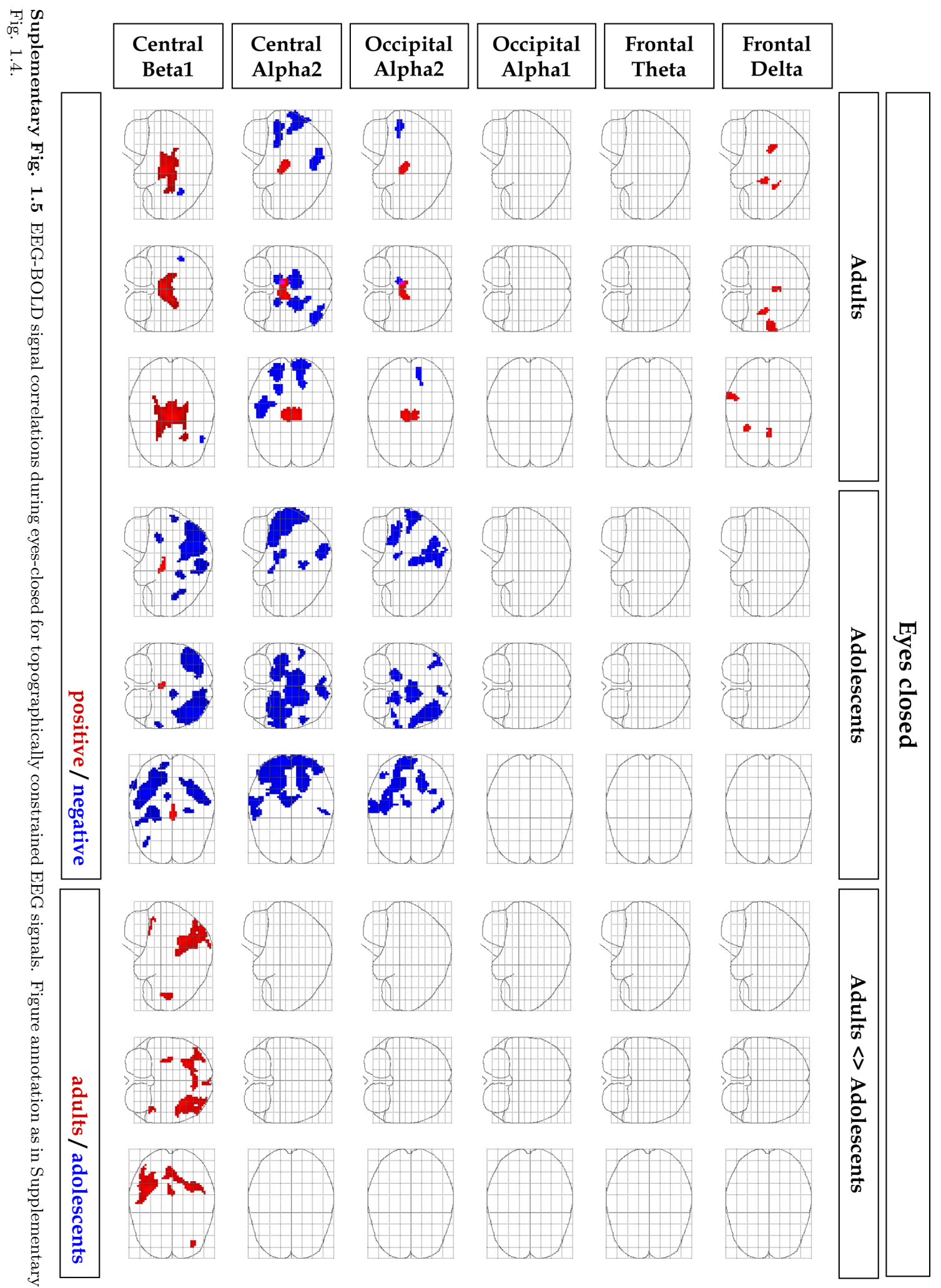

